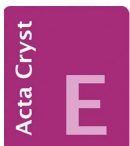

CRYSTALLOGRAPHIC COMMUNICATIONS

ISSN 2056-9890

Received 15 October 2018

Accepted 11 December 2018

Edited by C. Rizzoli, Universita degli Studi di Parma, Italy

Keywords: Caesalpinia pulcherrima; cassanetype diterpenoids; pulcherrimin C; Hirshfeld surface analysis; electrostatic potential; crystal structure.

CCDC reference: 1884284

Supporting information: this article has supporting information at journals.iucr.org/e

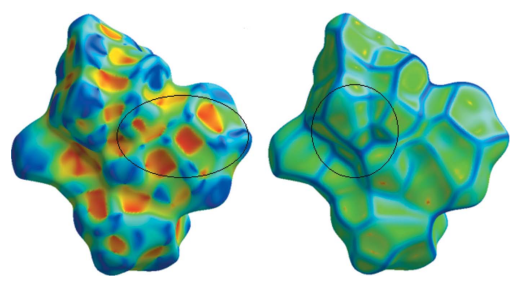

OPEN $\odot$ ACCESS

\section{Crystal structure, Hirshfeld surface analysis and electrostatic potential study of naturally occurring cassane-type diterpenoid Pulcherrimin C monohydrate at $100 \mathrm{~K}$}

\author{
Rajesh Kumar, ${ }^{a}$ K. Osahon Ogbeide, ${ }^{\mathrm{b}}$ Mujeeb-Ur-Rehman, ${ }^{\mathrm{a}}$ Bodunde Owolabi, \\ Abiodun Falodun, ${ }^{d}$ M. Iqbal Choudhary ${ }^{a}$ and Sammer Yousuf ${ }^{a *}$
}

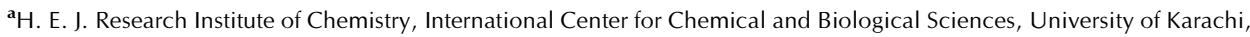
Karachi-75270, Pakistan, 'b Department of Chemistry, Faculty of Physical Sciences, University of Benin, Benin City, Nigeria, 'Department of Chemistry, School of Sciences, The Federal University of Technology, Akure, Nigeria, and ${ }^{\mathbf{d}}$ Department of Pharmaceutical Chemistry, Faculty of Pharmacy, University of Benin, Benin City, Nigeria.

*Correspondence e-mail: dr.sammer.yousuf@gmail.com

The title cassane-type diterpenoid known as pulcherrimin $\mathrm{C}, \mathrm{C}_{34} \mathrm{H}_{36} \mathrm{O}_{8} \cdot \mathrm{H}_{2} \mathrm{O}$, systematic name 5,6-bis(benzoyloxy)-4a-hydroxy-4,7,11b-trimethyl1,2,3,4,4a,5,6,6a,7,11,11a,11b-dodecahydrophenanthro[3,2-b]furan-4-carboxylic acid monohydrate, was isolated as a monohydrate from the medicinally important plant Caesalpinia pulcherrima, found in the tropical regions of south and south-east Asia. The molecule is composed of three trans-fused sixmembered rings having chair, chair and half-chair conformations, and a fivemembered planar furan ring. In the crystal, $\mathrm{O}-\mathrm{H} \cdots \mathrm{O}$ hydrogen bonds link molecules into chains parallel to the $b$ axis. Weak $\mathrm{C}-\mathrm{H} \cdots \pi$ interactions are also observed. Hirshfeld surface analysis indicates that the contribution of $\mathrm{O} \cdots \mathrm{H}$ interactions towards the total generated Hirshfeld surface is $21.5 \%$.

\section{Chemical context}

Caesalpinia pulcherrima (L) Swartz is an enduring shrub or small tree of the cassane family found in tropical regions of south and south-east Asia. It has been used ornamentally for a long time and is commonly known as Paradise flowers, Pride of Barbados and Peacock flower (Quisumbing, 1951). In addition, its parts have also been utilized as a traditional medicine in Thailand. The flowers and leaves are believed to be a cure for fever (Lotschert et al., 1983), and people in the northern regions of Thailand use its roots to treat tuberculous symptoms (Wutthithammaweach et al., 1997). Furthermore, it has also been proved that its crude DCM extract exhibits relatively strong anti-tubercular activity (Promsawan et al., 2003). A methanol extract of $C$. pulcherrima has been reported to have strong antibacterial activity (Parekh et al., 2006). The plant is also used to treat cardiovascular disorders, inflammation, muscular and sore pain, earache, and is known for its antipyretic, vermifugal and antimalarial activities (Patel et al., 2010; Roach et al., 2003). The present investigation deals with the isolation, single-crystal X-ray diffraction study, Hirshfeld surface analysis and electrostatic potential studies of the naturally occurring title compound, which was isolated as a monohydrate. 


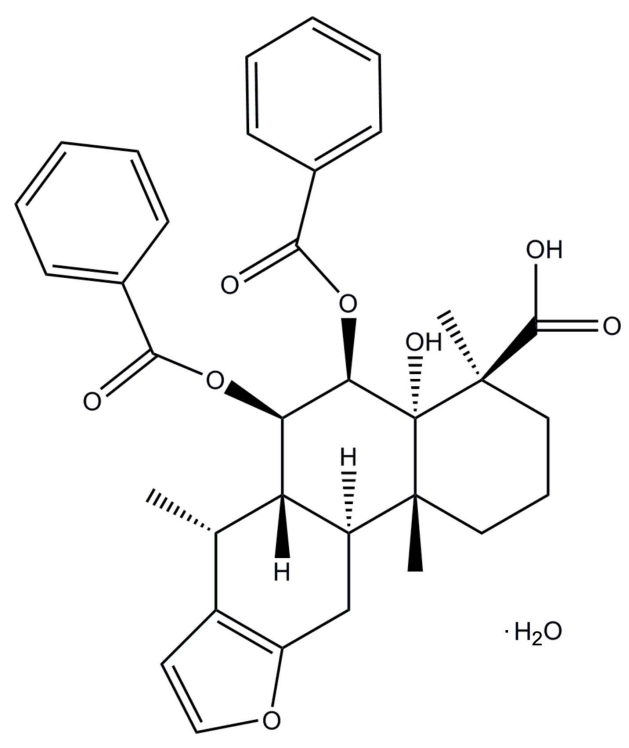

\section{Structural commentary}

The molecule of the title compound (Fig. 1) consists of three trans-fused rings, $A(\mathrm{C} 1-\mathrm{C} 5 / \mathrm{C} 10), B(\mathrm{C} 5-\mathrm{C} 10)$ and $C(\mathrm{C} 8 / \mathrm{C} 9 /$ C11-C14) having chair, chair and half-chair confirmations; the puckering parameters are $Q=0.554$ (3) $\AA, \theta=6.9(3)^{\circ}, \varphi=$ $6(3)^{\circ}$ for $A ; Q=0.591(3) \AA, \theta=0.0(3)^{\circ}, \varphi=318(12)^{\circ}$ for $B ; Q$

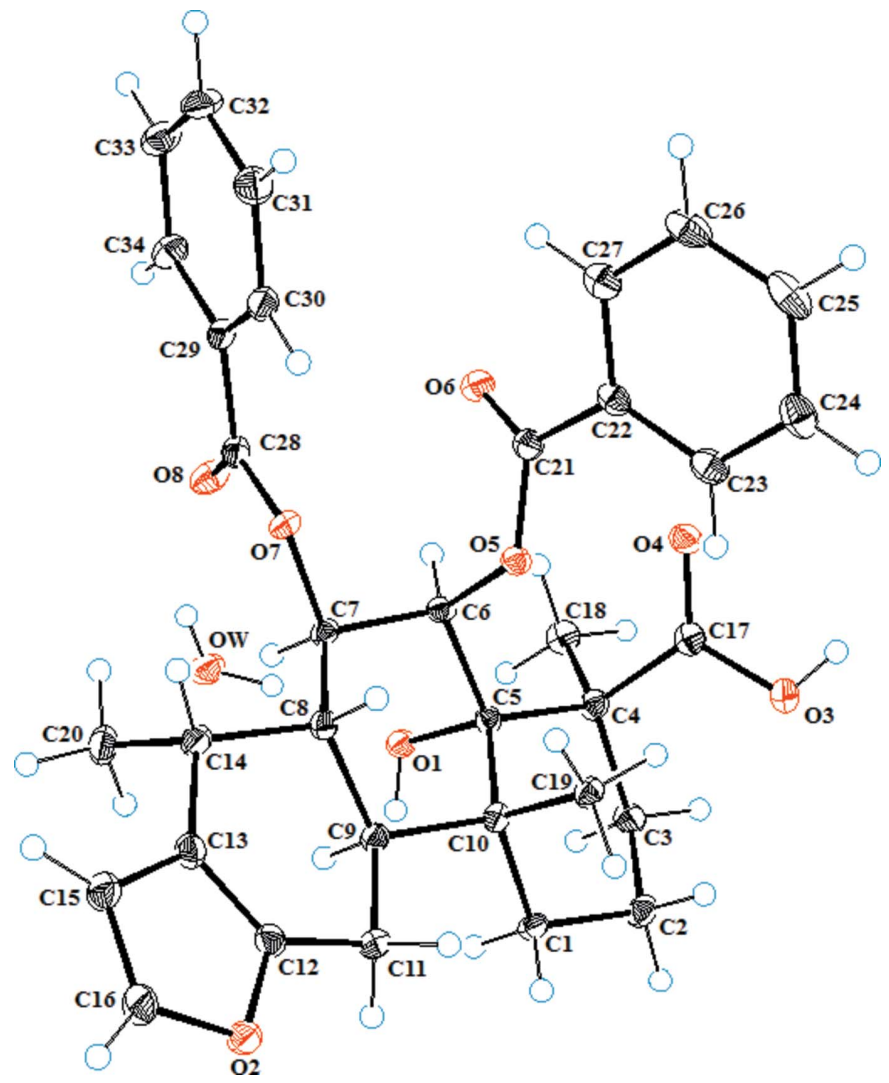

Figure 1

The molecular structure of the title compound with displacement ellipsoids drawn at the $50 \%$ probability level.
Table 1

Hydrogen-bond geometry $\left(\AA,^{\circ}\right)$.

$C g 1$ and $C g 2$ are the centroids of the $\mathrm{C} 29-\mathrm{C} 34$ and $\mathrm{C} 22-\mathrm{C} 27$ rings, respectively.

\begin{tabular}{lllll}
\hline$D-\mathrm{H} \cdots A$ & $D-\mathrm{H}$ & $\mathrm{H} \cdots A$ & $D \cdots A$ & $D-\mathrm{H} \cdots A$ \\
\hline $\mathrm{O} 1-\mathrm{H} 1 \cdots \mathrm{O} 4^{\mathrm{i}}$ & $0.82(4)$ & $2.01(4)$ & $2.654(3)$ & $134(4)$ \\
$\mathrm{O} 1 W-\mathrm{H} W A \cdots \mathrm{O} 1$ & $0.85(2)$ & $2.03(3)$ & $2.838(3)$ & $159(2)$ \\
$\mathrm{O} 1 W-\mathrm{H} W B \cdots \mathrm{O} 8$ & $0.85(2)$ & $2.27(3)$ & $3.062(3)$ & $154(2)$ \\
$\mathrm{O} 3-\mathrm{H} 3 \cdots \mathrm{O} 1 W^{\mathrm{ii}}$ & $0.83(4)$ & $1.86(4)$ & $2.680(3)$ & $174(4)$ \\
$\mathrm{C} 19-\mathrm{H} 19 B \cdots \mathrm{O} 3$ & 0.98 & 2.51 & $3.445(3)$ & 159 \\
$\mathrm{C} 1-\mathrm{H} 1 A \cdots \mathrm{Cg} 1^{\text {iii }}$ & 0.99 & 2.98 & $3.910(3)$ & 157 \\
$\mathrm{C} 34-\mathrm{H} 34 \cdots \mathrm{Cg} 2^{\mathrm{iv}}$ & 0.95 & 2.85 & $3.655(3)$ & 143 \\
\hline \multicolumn{2}{l}{ Symmetry codes: (i) } & $-x+1, y-\frac{1}{2},-z+\frac{3}{2} ;$ & (ii) & $-x+1, y+\frac{1}{2},-z+\frac{3}{2} ; \quad$ (iii) \\
$-x+\frac{1}{2},-y+1, z+\frac{1}{2} ;$ (iv) $x+\frac{1}{2},-y+\frac{3}{2},-z+1$. & &
\end{tabular}

$=0.446(3) \AA, \theta=48.0(4)^{\circ}, \varphi=12.4(5)^{\circ}$ for $C$. The adjacent cinnamoyl groups attached to atoms $\mathrm{C} 6$ and $\mathrm{C} 7$ are cis to each other, and the dihedral angle formed by their phenyl rings is $28.13(10)^{\circ}$. The planar furan ring $(\mathrm{O} 2 / \mathrm{C} 12 / \mathrm{C} 13 / \mathrm{C} 15 / \mathrm{C} 16)$ forms dihedral angles of $88.58(8)^{\circ}$ and $69.34(10)^{\circ}$, respectively, with the $\mathrm{C} 22-\mathrm{C} 27$ and $\mathrm{C} 29-\mathrm{C} 34$ phenyl rings. The absolute configurations of the stereogenic centers at atoms $\mathrm{C} 4$, $\mathrm{C} 5, \mathrm{C} 6, \mathrm{C} 7, \mathrm{C} 8, \mathrm{C} 9, \mathrm{C} 10$ and $\mathrm{C} 14$ are established as $S, S, R, R$, $R, S, R$ and $R$ on the basis of the reported literature (Patil et al., 1997).

The intramolecular $\mathrm{C} 19-\mathrm{H} 19 B \cdots \mathrm{O} 3$ hydrogen bond (Table 1) forms a ring with an $S(7)$ graph-set motif.

\section{Superamolecular features and Hirshfeld surface analysis}

Inter- and intramolecular interactions exert a significant influence on the geometry and properties of crystalline materials (Ferenczy et al., 2001; Putz et al., 2016). Analysis of the hydrogen bonding shows the presence of both conven-

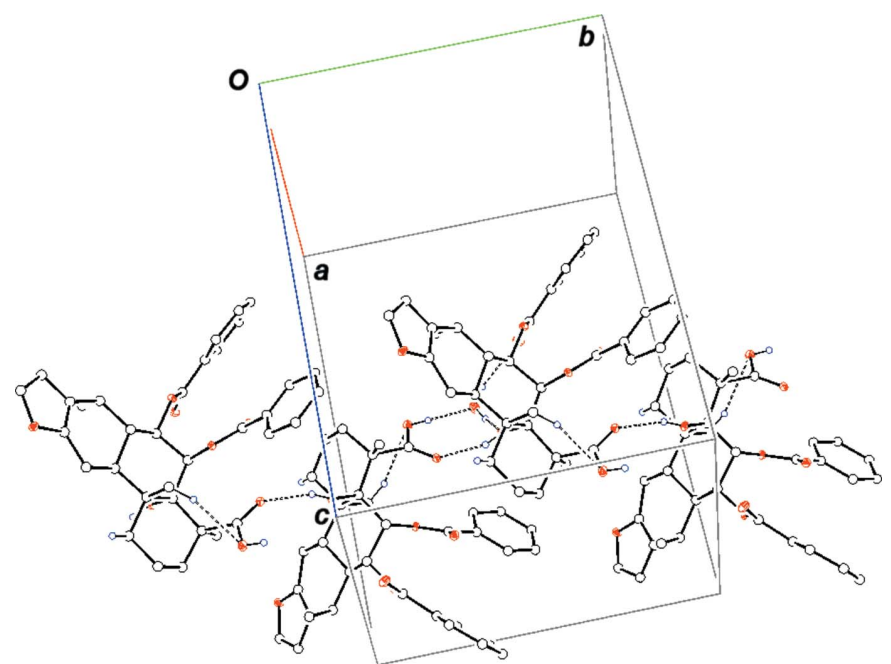

Figure 2

Partial packing diagram of the title compound showing the formation of a chain parallel to the $b$ axis by $\mathrm{O}-\mathrm{H} \cdots \mathrm{O}$ hydrogen bonds (dotted lines). Intramolecular $\mathrm{C}-\mathrm{H} \cdots \mathrm{O}$ hydrogen bonds (dotted lines) are also shown. Hydrogen atoms not involved in hydrogen bonding are omitted. 

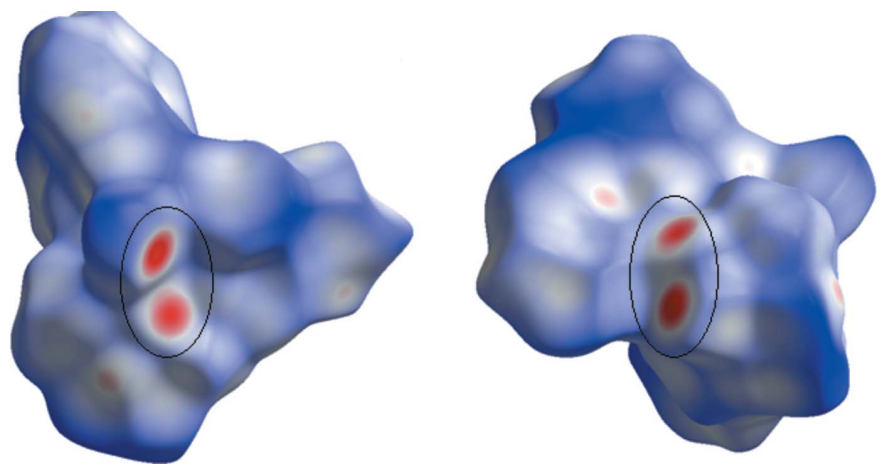

Figure 3

Hirshfeld surface mapped over $d_{\text {norm }}$ generated for the title compound.

tional and non-conventional types of hydrogen-bonded contacts in the crystal structure of the title compound (Fig. 2, Table 1). The oxygen atom of the water molecule acts as acceptor for the hydroxyl hydrogen atom of neighboring molecule via $\mathrm{O} 3-\mathrm{H} 3 \cdots \mathrm{O} 1 \mathrm{~W}$ interactions, while the two hydrogens atoms interact with the hydroxyl group at atom C5 and the carbonyl functionality of neighbouring molecules via $\mathrm{O} 1 W-\mathrm{H} W A \cdot \cdots \mathrm{O} 1$ and $\mathrm{O} 1 W-\mathrm{H} W B \cdots \mathrm{O} 8$ hydrogen bonds, forming an $R_{2}^{2}(10)$ ring. These interactions, along with the $\mathrm{O} 1-\mathrm{H} 1 \cdots \mathrm{O} 4$ hydrogen bond, link the molecules into chains parallel to the $b$ axis. Relatively weak $\mathrm{C}-\mathrm{H} \cdots \pi$ interactions (Table 1) are also observed.

The three-dimensional Hirshfeld surface calculated for the title compound is depicted in Fig. 3. The red regions indicate areas of close contacts shorter than the sum of van der Waals radii, while the blue and white regions represents contacts

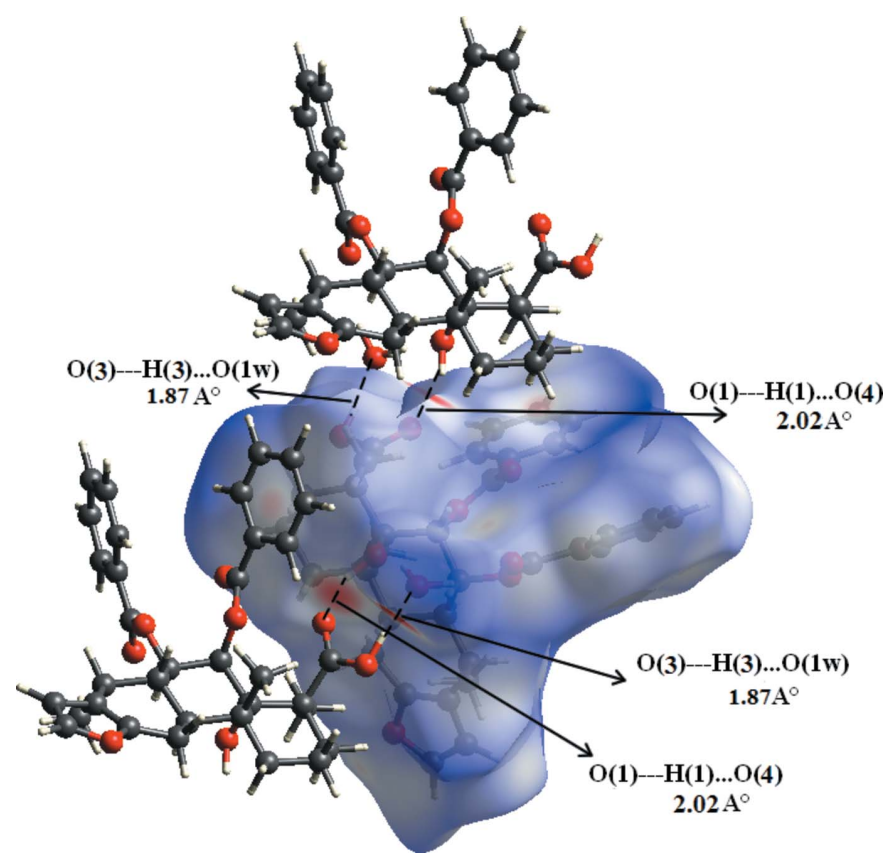

Figure 4

Hirshfeld surface mapped over $d_{\text {norm }}$ for the title compound with neighbouring molecules linked via $\mathrm{O}-\mathrm{H} \cdots \mathrm{O}$ hydrogen bonds (dashed lines).
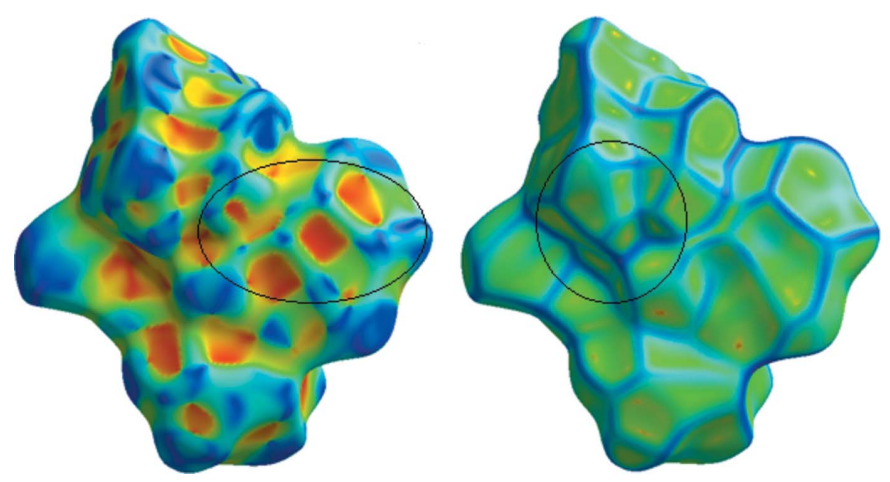

Figure 5

Hirshfeld surface mapped over shape-index for the title compound.

having distances greater and equal to the sum of van der Waals radii, respectively. The $\mathrm{O} 3-\mathrm{H} 3 \cdots \mathrm{O} 1 W$ and $\mathrm{O} 1-\mathrm{H} 1 \cdots \mathrm{O} 4$ hydrogen bonds are the two interactions responsible for linking neighboring molecules (Fig. 4). The curvedness surface (Fig. 5) shows the green (flat) and blue (curved) areas, representing low and high probabilities, respectively, of forming interactions with neighbouring molecules. The highlighted regions shown correspond to those in Fig. 3. No obvious adjacent blue or red triangles are present, indicating the absence of $\pi-\pi$ interactions. The fingerprint plots are presented in Fig. 6. H $\cdots \mathrm{H}$ contacts are the major contributor to the Hirshfeld surface $(58.1 \%)$. As a result of the presence of a water molecule in the asymmetric unit, $\mathrm{H} \cdots \mathrm{O}$ interactions are observed to contribute $21.5 \%$, with sharp spikes pointing toward the origin of the plot indicating the strength of the contacts. The contribution of $\mathrm{C} \cdots \mathrm{H}$ interactions is $17.5 \%$, whereas $\mathrm{C} \cdots \mathrm{O}$ interactions are negligible $(0.2 \%)$. The Hirshfeld surface mapped over electrostatic potential is shown in Fig. 7. The red regions indicate atoms with the potential to be hydrogen-bond acceptors (negative electrostatic potential), while blue regions indicate regions having atoms with positive electrostatic potential, i.e. hydrogen-bond donors.

\section{Database Survey}

A search of the Cambridge Structural Database (CSD version 5.39, update of August 2018; Groom et al., 2016) for a common fragment composed of three trans-fused six-membered rings and one planar furan ring gave 13 hits, including BEQVAX \{systematic name: $(4 \mathrm{a} R, 5 R, 6 R, 6 \mathrm{a} S, 7 R, 11 \mathrm{a} S, 11 \mathrm{~b} R)-4 \mathrm{a}, 6$-dihydroxy-4,4,7,11b-tetramethyl-1,2,3,4,4a,5,6,6a,7,11,11a,11b-dodecahydrophenanthro[3,2- $b$ ]furan-5-yl 3-phenylprop-2-enoate; Ogbeide et al., 2018), which has an $\alpha$-oriented methyl substituent at $\mathrm{C} 4$ and axially oriented cinnamoyl and hydroxyl substituents at C6 and C7. CSLPIN10 (1,2-desacetyl- $\varepsilon$ caesalpin 2-p-bromobenzoate; Birnbaum et al., 1969) is similar to the title compound but has different substituents at various positions including $\mathrm{C} 1$ and $\mathrm{C} 2$, with $\alpha$ - and $\beta$-oriented methyl substituents at C4 and C10. Refcode DUTJIM \{isovouacapenol C, \{systematic name: $(4 \mathrm{a} R, 5 R, 6 R, 6 \mathrm{a} S, 7 R, 11 \mathrm{a} S, 11 \mathrm{~b} R)$ 4a,6-dihydroxy-4,4,7,11b-tetramethyl-1,2,3,4,4a,5,6,6a,7,11,- 

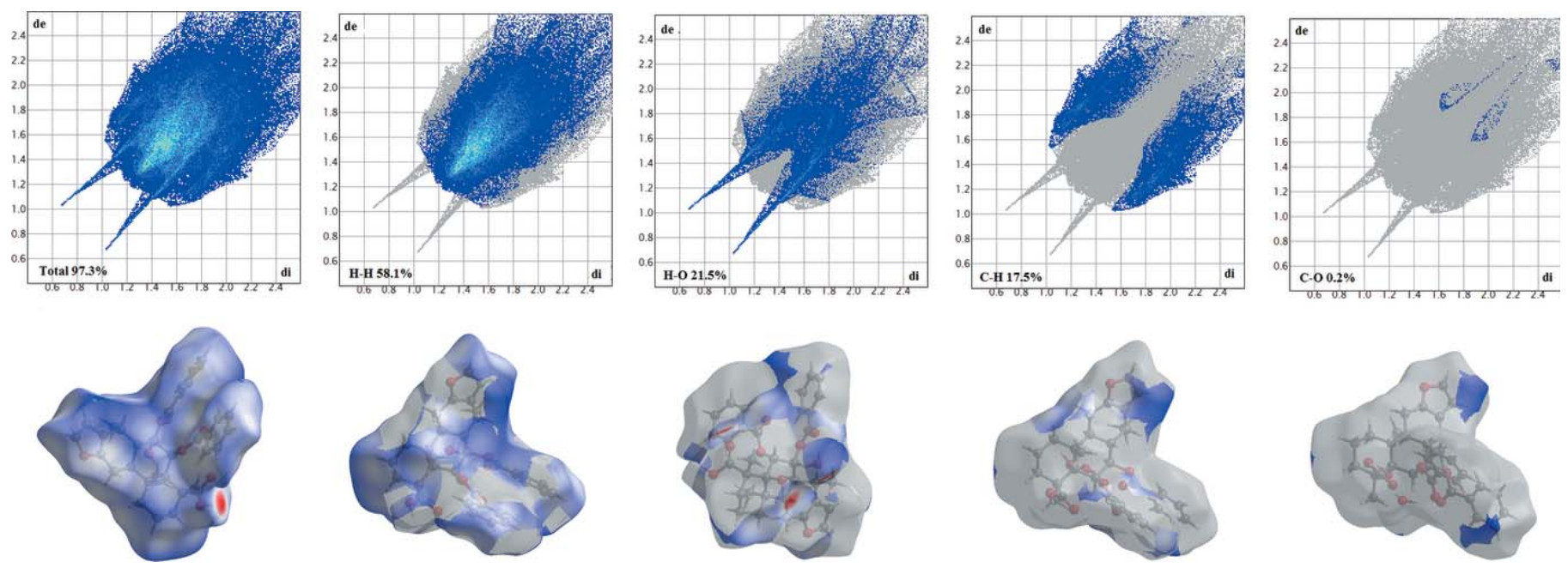

Figure 6

Two-dimensional fingerprint plots for the title compound together with areas of Hirshfeld surfaces involved in hydrogen bonding.

11a,11b-dodecahydrophenanthro[3,2-b]furan-5-yl benzoate and DUVCON \{vouacapen- $5 \alpha$-ol, systematic name: $(4 \mathrm{a} R, 6 \mathrm{a} S, 7 R, 11 \mathrm{a} S, 11 \mathrm{~b} R)-4,4,7,11 \mathrm{~b}$-tetramethyl-1,2,3,4,4a,5,6,6a, 7,11,11a,11b-dodecahydrophenanthro[3,2-b]furan-4a-ol\} were both also isolated from Caesalpinia pulcherrima (Fun et al., 2010) and show hydroxyl and benzoic acid substitution at C4 and C7, respectively. Compounds EGAYIU, EGAYUG, EGAZAN and EGAZER (Jiang et al., 2002), MEYREN, MEYRIR, MEYROX and MEYRUD (Jiang et al., 2001) and POPNIR (Kitagawa et al., 1994) all belong to the same class of
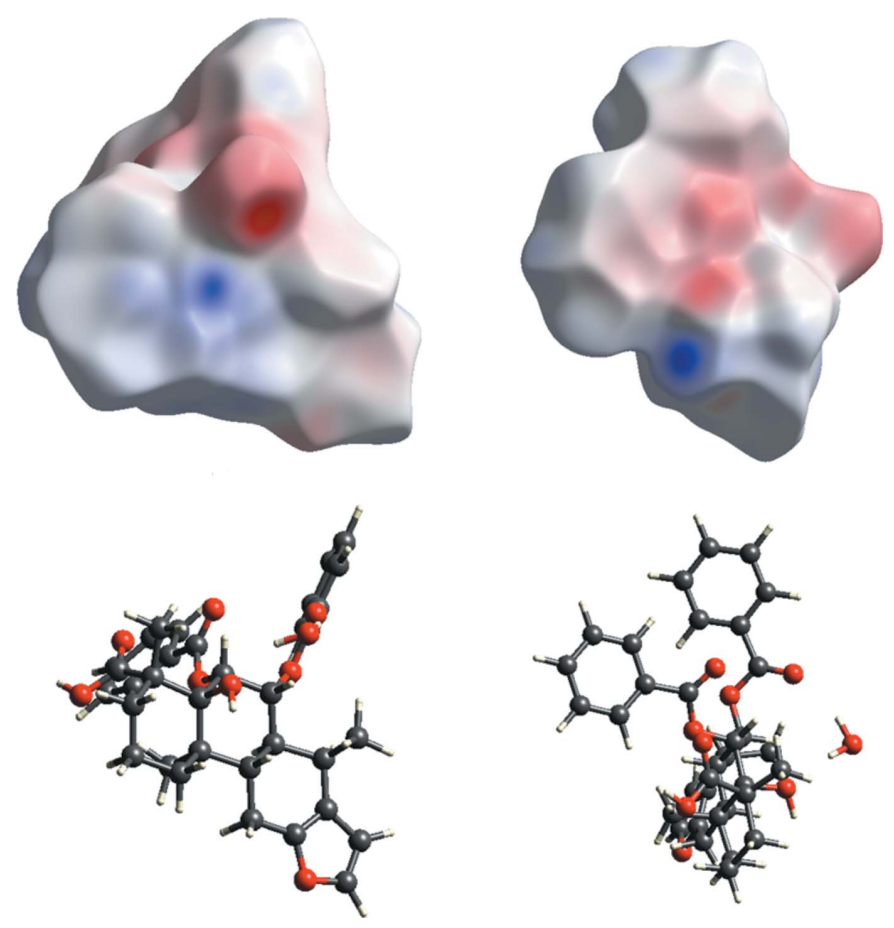

Figure 7

Electrostatic potential surface generated incorporated with the Hirshfeld surface for the title compound. compounds as the title compound, i.e. cassane-type diterpenoids, with different substitution patterns for the fused rings.

\section{Isolation and crystallization}

Fractions of the powdered stem bark of Caesalpinia pulcherrima were obtained according to the reported procedure (Ogbeide et al., 2018). Subfraction CP124-135 (755 mg) was chromatographed on silica gel $\left(\mathrm{SiO}_{2}, 2.5 \times 70 \mathrm{~cm}\right)$ and eluted isocratically with $20 \%$ ethylacetate in $n$-hexane to obtain a crystalline material, which was filtered and dried to give the purified title compound $(226 \mathrm{mg})$ known as pulcherrimin C. Crystals suitable for X-ray analysis were obtained by slow evaporation of an ethanol solution at $296 \mathrm{~K}$.

${ }^{1} \mathrm{H}$ NMR (400 MHz C $\left.{ }_{3} \mathrm{D}_{6} \mathrm{O}\right): 7.84(2 \mathrm{H}, m), 7.79(2 \mathrm{H}, m)$, $7.55(2 \mathrm{H}, m), 7.55(2 \mathrm{H}, m), 7.43(1 \mathrm{H}, m), 7.33(1 \mathrm{H}, m), 7.27$ $(1 \mathrm{H}, d, J=1.6 \mathrm{~Hz}), 6.21(1 \mathrm{H}, d, J=1.6 \mathrm{~Hz}), 6.18(1 \mathrm{H}, d, J=$ $3.6 \mathrm{~Hz}), 5.90(1 \mathrm{H}, b b, J=11.4 \mathrm{~Hz}, 3.8 \mathrm{~Hz}), 2.78(1 \mathrm{H}, m), 2.66$ $(1 \mathrm{H}, m), 2.59(1 \mathrm{H}, m), 2.46(1 \mathrm{H}, m), 2.31(1 \mathrm{H}, m), 1.50(1 \mathrm{H}, m)$, $1.93(1 \mathrm{H}, m), 1.62(1 \mathrm{H}, m), 1.89(1 \mathrm{H}, m), 1.79(2 \mathrm{H}, d, J=$ $13.6 \mathrm{~Hz}, 4.0 \mathrm{~Hz}), 1.57(3 \mathrm{H}, s), 1.41(3 \mathrm{H}, s), 0.99(3 \mathrm{H}, d, J=$ $6.8 \mathrm{~Hz})$. IR $\left(\mathrm{cm}^{-1}\right): 3527.7,2955.9,1718.4,1639.9,1456.7$, 1383.4, 1283.0, 1169.0, 1109.4, 1015.4, 966.5, 799.7, 715.1.

\section{Refinement}

Crystal data, data collection and structure refinement details are summarized in Table 2. The water $\mathrm{H}$ atoms were located in a difference-Fourier map and refined with the $\mathrm{O}-\mathrm{H}$ and $\mathrm{H} \cdots \mathrm{H}$ distances constrained to 0.85 (1) and 1.39 (1) $\AA$, respectively, and with $U_{\text {iso }}(\mathrm{H})=1.5 U_{\text {eq }}(\mathrm{O})$. All other $\mathrm{H}$ atoms were positioned with idealized geometry and refined isotropically with $\mathrm{O}-\mathrm{H}=0.83 \AA, \mathrm{C}-\mathrm{H}=0.95-1.00 \AA$, and with $U_{\text {iso }}(\mathrm{H})=$ $1.2 U_{\text {eq }}(\mathrm{C})$ or $1.5 U_{\text {eq }}(\mathrm{C}-m e t h y l, \mathrm{O})$. A rotating model was used for the methyl and hydroxy groups. 


\section{Funding information}

This research was supported by the University of Benin (URPC 2016 grant), The World Academy of Sciences (TWAS), the International Center for Chemical and Biological Sciences (ICCBS), University of Karachi, Pakistan (ICCBS-TWAS Postgraduate Fellowship Award to KOO; FR No. 3240287190) and the Higher Education Commission Pakistan (research grant No. 20-2830).

\section{References}

Birnbaum, K. B. \& Ferguson, G. (1969). Acta Cryst. B25, 720-730.

Bruker (2000). SADABS, SMART and SAINT. Bruker AXS Inc., Madison, Wisconsin, USA.

Ferenczy, G. G. \& Ángyán, J. G. (2001). J. Comput. Chem. 22, 16791690.

Fun, H.-K., Yodsaoue, O., Karalai, C. \& Chantrapromma, S. (2010). Acta Cryst. E66, o2059-o2060.

Groom, C. R., Bruno, I. J., Lightfoot, M. P. \& Ward, S. C. (2016). Acta Cryst. B72, 171-179.

Jiang, R. W., Ma, S. C., But, P. P. H. \& Mak, T. C. (2001). J. Nat. Prod. 64, 1266-1272.

Jiang, R. W., Ma, S. C., He, Z. D., Huang, X. S., But, P. P. H., Wang, H., Chan, S. P., Ooi, V. E., Xu, H. X. \& Mak, T. C. (2002). Bioorg. Med. Chem. 10, 2161-2170.

Kitagawa, I., Simanjuntak, P., Watano, T., Shibuya, H., Fujii, S., Yamagata, Y. \& Kobayashi, M. (1994). Chem. Pharm. Bull. 42, 1798-1802.

Lotschert, W. \& Beese, G. (1983). Collins Guide to Tropical Plants. London: Collins.

Ogbeide, K. O., Kumar, R., Mujeeb-Ur-Rehman, Owolabi, B., Falodun, A., Choudhary, M. I. \& Yousuf, S. (2018). Acta Cryst. E74, 385-389.

Parekh, J., Jadeja, D. \& Chanda, S. (2006). Turk. J. Biol. 29, 203-210.

Parsons, S., Flack, H. D. \& Wagner, T. (2013). Acta Cryst. B69, 249259.

Patel, S. S., Verma, N. K., Chatterjee, C. \& Gauthaman, K. (2010). Int. J. Appl. Res. Nat. Prod. 3, 1-5.

Patil, A. D., Freyer, A. J., Lee Webb, R., Zuber, G., Reichwein, R., Bean, M. F., Faucette, L. \& Johnson, R. K. (1997). Tetrahedron, 53, 1583-1592.

Promsawan, N., Kittakoop, P., Boonphong, S. \& Nongkunsarn, P. (2003). Planta Med. 69, 776-777.

Putz, M. V., Duda-Seiman, C., Duda-Seiman, D., Putz, A., Alexandrescu, I., Mernea, M. \& Avram, S. (2016). Int. J. Mol. Sci. 17, 1087.
Table 2

Experimental details.

\begin{tabular}{ll}
\hline Crystal data & \\
Chemical formula & $\mathrm{C}_{34} \mathrm{H}_{36} \mathrm{O}_{8} \cdot \mathrm{H}_{2} \mathrm{O}$ \\
$M_{\mathrm{r}}$ & 590.64 \\
Crystal system, space group & Orthorhombic, $P 2_{1} 2_{1} 2_{1}$ \\
Temperature $(\mathrm{K})$ & 100 \\
$a, b, c(\AA)$ & $11.8027(7), 13.2843(8)$, \\
$V\left(\AA^{3}\right)$ & $19.0835(10)$ \\
$Z$ & $2992.1(3)$ \\
Radiation type & 4 \\
$\mu\left(\mathrm{mm}^{-1}\right)$ & $\mathrm{Cu}$ K $\alpha$ \\
Crystal size $(\mathrm{mm})$ & 0.78 \\
& $0.35 \times 0.24 \times 0.10$ \\
Data collection & \\
Diffractometer & Bruker APEXII CCD \\
Absorption correction & Multi-scan $(S A D A B S ;$ Bruker, \\
& $2000)$ \\
$T_{\text {min }}, T_{\text {max }}$ & $0.772,0.926$ \\
No. of measured, independent and & $18780,5425,4931$ \\
$\quad$ observed $[I>2 \sigma(I)]$ reflections & \\
$R_{\text {int }}$ & 0.061 \\
$(\text { sin } \theta / \lambda)_{\text {max }}\left(\AA^{-1}\right)$ & 0.602 \\
& \\
Refinement & \\
$R\left[F^{2}>2 \sigma\left(F^{2}\right)\right], w R\left(F^{2}\right), S$ & $0.040,0.098,1.03$ \\
No. of reflections & 5425 \\
No. of parameters & 401 \\
No. of restraints & 3 \\
H-atom treatment & H atoms treated by a mixture of \\
& independent and constrained \\
$\Delta \rho_{\text {max }}, \Delta \rho_{\text {min }}\left(\mathrm{e} \AA^{-3}\right)$ & refinement \\
Absolute structure & $0.20,-0.23$ \\
& Flack $x$ determined using 1939 \\
Absolute structure parameter & $0.02(9)$ \\
\hline &
\end{tabular}

Computer programs: APEX2 and SAINT (Bruker, 2000), SHELXT2014 (Sheldrick, 2015a), SHELXL2018 (Sheldrick, 2015b) and SHELXTL (Sheldrick, 2008).

Quisumbing, E. A. (1951). Medicinal Plants of the Philippines. Manila: Department of Agriculture and Commerce.

Roach, J. S., McLean, S., Reynolds, W. F. \& Tinto, W. F. (2003). J. Nat. Prod. 66, 1378-1381.

Sheldrick, G. M. (2008). Acta Cryst. A64, 112-122.

Sheldrick, G. M. (2015a). Acta Cryst. A71, 3-8.

Sheldrick, G. M. (2015b). Acta Cryst. C71, 3-8.

Wutthithammaweach, W. (1997). Encyclopedia of Herbs: Compilation of Thai Pharmaceuticals. Bangkok: Odion Store. 


\section{supporting information}

Acta Cryst. (2019). E75, 119-123 [https://doi.org/10.1107/S2056989018017498]

Crystal structure, Hirshfeld surface analysis and electrostatic potential study of naturally occurring cassane-type diterpenoid Pulcherrimin C monohydrate at $100 \mathrm{~K}$

Rajesh Kumar, K. Osahon Ogbeide, Mujeeb-Ur-Rehman, Bodunde Owolabi, Abiodun Falodun, M. Iqbal Choudhary and Sammer Yousuf

Computing details

Data collection: APEX2 (Bruker, 2000); cell refinement: SAINT (Bruker, 2000); data reduction: SAINT (Bruker, 2000); program(s) used to solve structure: SHELXT2014 (Sheldrick, 2015a); program(s) used to refine structure: SHELXL2018 (Sheldrick, 2015b); molecular graphics: SHELXTL (Sheldrick, 2008); software used to prepare material for publication: SHELXTL (Sheldrick, 2008).

5,6-Bis(benzoyloxy)-4a-hydroxy-4,7,11 b-trimethyl-1,2,3,4,4a,5,6,6a,7,11,11a, 11 b-dodecahydrophenanthro[3,2b] furan-4-carboxylic acid monohydrate

Crystal data

$\mathrm{C}_{34} \mathrm{H}_{36} \mathrm{O}_{8} \cdot \mathrm{H}_{2} \mathrm{O}$

$M_{r}=590.64$

Orthorhombic, $P 2_{1} 2_{1} 2_{1}$

$a=11.8027$ (7) $\AA$

$b=13.2843(8) \AA$

$c=19.0835(10) \AA$

$V=2992.1(3) \AA^{3}$

$Z=4$

$F(000)=1256$

Data collection

Bruker APEXII CCD

diffractometer

$\varphi$ and $\omega$ scans

Absorption correction: multi-scan

(SADABS; Bruker, 2000)

$T_{\min }=0.772, T_{\max }=0.926$

18780 measured reflections

\section{Refinement}

Refinement on $F^{2}$

Least-squares matrix: full

$R\left[F^{2}>2 \sigma\left(F^{2}\right)\right]=0.040$

$w R\left(F^{2}\right)=0.098$

$S=1.03$

5425 reflections
$D_{\mathrm{x}}=1.311 \mathrm{Mg} \mathrm{m}^{-3}$

$\mathrm{Cu} K \alpha$ radiation, $\lambda=1.54178 \AA$

Cell parameters from 9847 reflections

$\theta=4.1-68.2^{\circ}$

$\mu=0.78 \mathrm{~mm}^{-1}$

$T=100 \mathrm{~K}$

Block, colourless

$0.35 \times 0.24 \times 0.10 \mathrm{~mm}$

5425 independent reflections

4931 reflections with $I>2 \sigma(I)$

$R_{\text {int }}=0.061$

$\theta_{\text {max }}=68.2^{\circ}, \theta_{\text {min }}=4.1^{\circ}$

$h=-14 \rightarrow 14$

$k=-12 \rightarrow 16$

$l=-22 \rightarrow 22$

401 parameters

3 restraints

Hydrogen site location: mixed

$\mathrm{H}$ atoms treated by a mixture of independent and constrained refinement 
$w=1 /\left[\sigma^{2}\left(F_{\mathrm{o}}^{2}\right)+(0.0537 P)^{2}+0.4392 P\right]$

where $P=\left(F_{\mathrm{o}}^{2}+2 F_{\mathrm{c}}^{2}\right) / 3$

$(\Delta / \sigma)_{\max }<0.001$

$\Delta \rho_{\max }=0.20 \mathrm{e} \AA^{-3}$

$\Delta \rho_{\min }=-0.23$ e $\AA^{-3}$

Special details

Geometry. All esds (except the esd in the dihedral angle between two 1.s. planes) are estimated using the full covariance matrix. The cell esds are taken into account individually in the estimation of esds in distances, angles and torsion angles; correlations between esds in cell parameters are only used when they are defined by crystal symmetry. An approximate (isotropic) treatment of cell esds is used for estimating esds involving l.s. planes.

Fractional atomic coordinates and isotropic or equivalent isotropic displacement parameters $\left(\AA^{2}\right)$

\begin{tabular}{|c|c|c|c|c|}
\hline & $x$ & $y$ & $z$ & $U_{\text {iso }} * / U_{\text {eq }}$ \\
\hline $\mathrm{O} 1$ & $0.50438(17)$ & $0.47639(16)$ & $0.71403(10)$ & $0.0169(4)$ \\
\hline H1 & $0.498(2)$ & $0.423(3)$ & $0.7356(16)$ & $0.025^{*}$ \\
\hline $\mathrm{O} 2$ & $0.01638(17)$ & $0.25183(15)$ & $0.67636(10)$ & $0.0208(4)$ \\
\hline $\mathrm{O} 1 \mathrm{~W}$ & $0.63748(19)$ & $0.41758(16)$ & $0.59717(11)$ & $0.0264(5)$ \\
\hline HWA & $0.614(3)$ & $0.442(2)$ & $0.6356(7)$ & $0.040^{*}$ \\
\hline HWB & $0.610(3)$ & $0.451(2)$ & $0.5630(8)$ & $0.040^{*}$ \\
\hline $\mathrm{O} 3$ & 0.3949 (2) & $0.72018(17)$ & $0.88174(10)$ & $0.0244(5)$ \\
\hline H3 & $0.381(3)$ & $0.780(3)$ & $0.8896(9)$ & $0.037 *$ \\
\hline $\mathrm{O} 4$ & $0.44345(18)$ & $0.78239(15)$ & $0.77797(10)$ & $0.0222(5)$ \\
\hline O5 & $0.31651(16)$ & $0.68572(14)$ & $0.68136(9)$ & $0.0153(4)$ \\
\hline O6 & $0.41856(17)$ & $0.78789(15)$ & $0.61026(10)$ & $0.0196(4)$ \\
\hline $\mathrm{O} 7$ & $0.32786(16)$ & $0.59542(15)$ & $0.55359(9)$ & $0.0164(4)$ \\
\hline O8 & $0.50711(18)$ & $0.57755(17)$ & $0.51448(10)$ & $0.0243(5)$ \\
\hline $\mathrm{C} 1$ & $0.3445(2)$ & $0.4239(2)$ & $0.82933(13)$ & $0.0169(6)$ \\
\hline $\mathrm{H} 1 \mathrm{~A}$ & 0.275590 & 0.389055 & 0.846246 & $0.020^{*}$ \\
\hline H1B & 0.399822 & 0.371907 & 0.814829 & $0.020^{*}$ \\
\hline $\mathrm{C} 2$ & $0.3947(3)$ & $0.4855(2)$ & $0.88895(14)$ & $0.0200(6)$ \\
\hline $\mathrm{H} 2 \mathrm{~A}$ & 0.413298 & 0.440659 & 0.928742 & $0.024 *$ \\
\hline $\mathrm{H} 2 \mathrm{~B}$ & 0.338465 & 0.535594 & 0.905294 & $0.024^{*}$ \\
\hline $\mathrm{C} 3$ & $0.5013(3)$ & $0.5393(2)$ & $0.86435(14)$ & $0.0191(6)$ \\
\hline $\mathrm{H} 3 \mathrm{~A}$ & 0.559558 & 0.487970 & 0.853582 & $0.023 *$ \\
\hline H3B & 0.530399 & 0.580736 & 0.903561 & $0.023^{*}$ \\
\hline $\mathrm{C} 4$ & $0.4874(2)$ & $0.6082(2)$ & $0.79929(14)$ & $0.0173(6)$ \\
\hline $\mathrm{C} 5$ & $0.4224(2)$ & $0.5473(2)$ & $0.73970(14)$ & $0.0143(6)$ \\
\hline C6 & $0.3989(2)$ & $0.6071(2)$ & $0.67137(14)$ & $0.0147(6)$ \\
\hline H6 & 0.471384 & 0.637824 & 0.654684 & $0.018^{*}$ \\
\hline $\mathrm{C} 7$ & $0.3546(2)$ & $0.5350(2)$ & $0.61502(14)$ & $0.0150(6)$ \\
\hline $\mathrm{H} 7$ & 0.415279 & 0.485543 & 0.602790 & $0.018^{*}$ \\
\hline $\mathrm{C} 8$ & $0.2478(3)$ & $0.4785(2)$ & $0.63531(13)$ & $0.0155(6)$ \\
\hline $\mathrm{H} 8$ & 0.186764 & 0.528923 & 0.644741 & $0.019 *$ \\
\hline C9 & $0.2713(2)$ & $0.4192(2)$ & $0.70451(13)$ & $0.0155(6)$ \\
\hline H9 & 0.334191 & 0.370940 & 0.694319 & $0.019 *$ \\
\hline $\mathrm{C} 10$ & $0.3135(2)$ & $0.4897(2)$ & $0.76501(14)$ & $0.0148(6)$ \\
\hline C11 & $0.1683(2)$ & 0.3559 (2) & $0.72822(14)$ & $0.0179(6)$ \\
\hline
\end{tabular}

Absolute structure: Flack $x$ determined using 1939 quotients $\left[\left(I^{+}\right)-\left(I^{-}\right)\right] /\left[\left(I^{+}\right)+\left(I^{-}\right)\right]$(Parsons et al., 2013)

Absolute structure parameter: $0.02(9)$ 


\begin{tabular}{|c|c|c|c|c|}
\hline H11A & 0.194840 & 0.298367 & 0.756862 & $0.021^{*}$ \\
\hline H11B & 0.117878 & 0.397728 & 0.757681 & $0.021^{*}$ \\
\hline $\mathrm{C} 12$ & $0.1045(3)$ & $0.3179(2)$ & $0.66718(15)$ & $0.0178(6)$ \\
\hline $\mathrm{C} 13$ & $0.1173(3)$ & $0.3412(2)$ & $0.59897(15)$ & $0.0188(6)$ \\
\hline $\mathrm{C} 14$ & $0.2088(2)$ & $0.4101(2)$ & $0.57349(14)$ & $0.0182(6)$ \\
\hline H14 & 0.175084 & 0.454722 & 0.536778 & $0.022 *$ \\
\hline C15 & $0.0311(3)$ & $0.2860(2)$ & $0.56192(15)$ & $0.0232(7)$ \\
\hline H15 & 0.017844 & 0.286084 & 0.512817 & $0.028^{*}$ \\
\hline $\mathrm{C} 16$ & $-0.0266(3)$ & $0.2344(2)$ & $0.61042(15)$ & $0.0239(7)$ \\
\hline H16 & -0.088919 & 0.191433 & 0.600640 & $0.029^{*}$ \\
\hline $\mathrm{C} 17$ & $0.4377(2)$ & 0.7109 (2) & 0.81719 (14) & $0.0176(6)$ \\
\hline $\mathrm{C} 18$ & $0.6083(2)$ & $0.6352(2)$ & $0.77395(15)$ & $0.0210(6)$ \\
\hline H18A & 0.649574 & 0.573260 & 0.762598 & $0.031 *$ \\
\hline H18B & 0.648405 & 0.671535 & 0.811094 & $0.031^{*}$ \\
\hline $\mathrm{H} 18 \mathrm{C}$ & 0.603385 & 0.677712 & 0.732109 & $0.031^{*}$ \\
\hline C19 & 0.2189 (2) & $0.5629(2)$ & $0.78820(14)$ & $0.0165(6)$ \\
\hline H19A & 0.178146 & 0.587521 & 0.746845 & $0.025^{*}$ \\
\hline H19B & 0.252518 & 0.619990 & 0.813222 & $0.025^{*}$ \\
\hline $\mathrm{H} 19 \mathrm{C}$ & 0.165902 & 0.527710 & 0.819244 & $0.025^{*}$ \\
\hline $\mathrm{C} 20$ & $0.3041(3)$ & $0.3486(2)$ & $0.53868(15)$ & $0.0258(7)$ \\
\hline $\mathrm{H} 20 \mathrm{~A}$ & 0.271281 & 0.302184 & 0.504307 & $0.039 *$ \\
\hline H20B & 0.344815 & 0.310097 & 0.574548 & $0.039^{*}$ \\
\hline $\mathrm{H} 20 \mathrm{C}$ & 0.356908 & 0.394307 & 0.515049 & $0.039^{*}$ \\
\hline $\mathrm{C} 21$ & $0.3382(2)$ & $0.7741(2)$ & $0.64816(14)$ & $0.0171(6)$ \\
\hline $\mathrm{C} 22$ & $0.2512(2)$ & $0.8514(2)$ & 0.66403 (14) & $0.0173(6)$ \\
\hline $\mathrm{C} 23$ & $0.1932(3)$ & $0.8533(2)$ & $0.72745(16)$ & $0.0218(6)$ \\
\hline $\mathrm{H} 23$ & 0.203683 & 0.800542 & 0.760439 & $0.026^{*}$ \\
\hline $\mathrm{C} 24$ & 0.1201 (3) & $0.9321(2)$ & $0.74262(17)$ & $0.0256(7)$ \\
\hline $\mathrm{H} 24$ & 0.081958 & 0.934114 & 0.786437 & $0.031^{*}$ \\
\hline $\mathrm{C} 25$ & 0.1025 (3) & $1.0083(2)$ & $0.69359(18)$ & $0.0275(7)$ \\
\hline $\mathrm{H} 25$ & 0.052511 & 1.062358 & 0.703969 & $0.033^{*}$ \\
\hline $\mathrm{C} 26$ & $0.1582(3)$ & $1.0050(2)$ & $0.62952(17)$ & $0.0253(7)$ \\
\hline H26 & 0.144897 & 1.056131 & 0.595677 & $0.030^{*}$ \\
\hline $\mathrm{C} 27$ & $0.2331(3)$ & $0.9277(2)$ & $0.61472(15)$ & $0.0216(6)$ \\
\hline $\mathrm{H} 27$ & 0.272173 & 0.926462 & 0.571194 & $0.026^{*}$ \\
\hline $\mathrm{C} 28$ & $0.4154(2)$ & $0.6172(2)$ & $0.51063(14)$ & $0.0175(6)$ \\
\hline $\mathrm{C} 29$ & 0.3839 (3) & 0.6969 (2) & $0.45916(14)$ & $0.0189(6)$ \\
\hline $\mathrm{C} 30$ & $0.2788(2)$ & $0.7442(2)$ & $0.46095(14)$ & $0.0194(6)$ \\
\hline $\mathrm{H} 30$ & 0.223119 & 0.723328 & 0.493792 & $0.023^{*}$ \\
\hline $\mathrm{C} 31$ & $0.2556(3)$ & $0.8222(2)$ & $0.41454(16)$ & $0.0256(7)$ \\
\hline H31 & 0.184156 & 0.855115 & 0.415744 & $0.031 *$ \\
\hline $\mathrm{C} 32$ & $0.3373(3)$ & 0.8517 (3) & $0.36640(16)$ & $0.0279(7)$ \\
\hline H32 & 0.321630 & 0.905285 & 0.334856 & $0.033^{*}$ \\
\hline $\mathrm{C} 33$ & $0.4411(3)$ & 0.8041 (3) & $0.36394(16)$ & $0.0267(7)$ \\
\hline H33 & 0.496239 & 0.824739 & 0.330637 & $0.032 *$ \\
\hline $\mathrm{C} 34$ & 0.4651 (3) & $0.7262(2)$ & $0.40992(15)$ & $0.0221(6)$ \\
\hline H34 & 0.536337 & 0.693019 & 0.407994 & $0.027^{*}$ \\
\hline
\end{tabular}


Atomic displacement parameters $\left(\AA^{2}\right)$

\begin{tabular}{|c|c|c|c|c|c|c|}
\hline & $U^{11}$ & $U^{22}$ & $U^{33}$ & $U^{12}$ & $U^{13}$ & $U^{23}$ \\
\hline O1 & $0.0187(11)$ & $0.0154(10)$ & $0.0167(9)$ & $0.0038(9)$ & $0.0027(8)$ & $0.0020(7)$ \\
\hline $\mathrm{O} 2$ & $0.0228(11)$ & $0.0219(11)$ & $0.0177(10)$ & $-0.0070(9)$ & $-0.0007(8)$ & $0.0001(8)$ \\
\hline $\mathrm{O} 1 \mathrm{~W}$ & $0.0306(13)$ & $0.0244(12)$ & $0.0243(10)$ & $0.0037(10)$ & $0.0089(9)$ & $0.0046(9)$ \\
\hline $\mathrm{O} 3$ & $0.0342(13)$ & 0.0197 (11) & $0.0194(10)$ & $0.0030(10)$ & $0.0037(9)$ & $-0.0026(8)$ \\
\hline $\mathrm{O} 4$ & 0.0307 (12) & $0.0169(10)$ & $0.0190(10)$ & $-0.0040(9)$ & $-0.0041(9)$ & $0.0005(8)$ \\
\hline O5 & $0.0152(10)$ & $0.0142(10)$ & $0.0166(9)$ & $0.0018(8)$ & $0.0008(8)$ & $0.0007(7)$ \\
\hline O6 & 0.0203 (11) & $0.0204(11)$ & $0.0180(9)$ & $-0.0015(9)$ & $0.0015(8)$ & $0.0023(8)$ \\
\hline O7 & $0.0170(10)$ & $0.0190(10)$ & $0.0131(9)$ & $-0.0003(9)$ & $0.0025(8)$ & $0.0030(7)$ \\
\hline O8 & $0.0216(11)$ & $0.0305(12)$ & 0.0207 (10) & $0.0051(10)$ & $0.0040(9)$ & $0.0061(9)$ \\
\hline $\mathrm{C} 1$ & $0.0196(14)$ & $0.0167(14)$ & 0.0143 (12) & 0.0003 (12) & $0.0023(11)$ & $0.0027(10)$ \\
\hline $\mathrm{C} 2$ & $0.0245(16)$ & $0.0222(15)$ & 0.0133 (13) & $0.0031(13)$ & $-0.0010(11)$ & $0.0018(11)$ \\
\hline C3 & 0.0223 (16) & $0.0172(15)$ & 0.0178 (13) & $0.0023(13)$ & $-0.0059(12)$ & $0.0015(11)$ \\
\hline $\mathrm{C} 4$ & $0.0179(15)$ & $0.0192(15)$ & 0.0148 (13) & $-0.0021(13)$ & $-0.0019(11)$ & $-0.0012(10)$ \\
\hline $\mathrm{C} 5$ & $0.0163(14)$ & $0.0135(14)$ & $0.0132(12)$ & $0.0019(12)$ & $-0.0012(11)$ & $-0.0007(10)$ \\
\hline C6 & $0.0141(13)$ & $0.0141(13)$ & $0.0158(12)$ & $0.0001(12)$ & $0.0025(11)$ & $0.0025(10)$ \\
\hline $\mathrm{C} 7$ & $0.0177(15)$ & $0.0152(14)$ & $0.0120(12)$ & $0.0036(12)$ & 0.0005 & $0.0020(10)$ \\
\hline $\mathrm{C} 8$ & $0.0168(14)$ & $0.0152(14)$ & $0.0144(12)$ & $0.0016(12)$ & $0.0017(11)$ & $-0.0002(10)$ \\
\hline $\mathrm{C} 9$ & $0.0171(14)$ & $0.0153(14)$ & $0.0140(12)$ & $0.0016(12)$ & $0.0020(10)$ & $0.0006(10)$ \\
\hline $\mathrm{C} 10$ & $0.0151(14)$ & $0.0166(14)$ & $0.0127(12)$ & $0.0013(12)$ & $-0.0001(11)$ & $-0.0008(10)$ \\
\hline $\mathrm{C} 11$ & $0.0200(15)$ & $0.0180(14)$ & $0.0156(13)$ & $-0.0021(12)$ & $0.0032(12)$ & $0.0013(11)$ \\
\hline $\mathrm{C} 12$ & $0.0187(15)$ & $0.0150(13)$ & 0.0197 (13) & $0.0001(12)$ & $0.0038(12)$ & $-0.0023(11)$ \\
\hline $\mathrm{C} 13$ & 0.0209 (16) & $0.0181(15)$ & $0.0174(13)$ & $-0.0008(13)$ & $-0.0018(12)$ & $-0.0025(11)$ \\
\hline $\mathrm{C} 14$ & $0.0205(15)$ & $0.0200(15)$ & $0.0140(12)$ & $-0.0032(13)$ & $0.0002(11)$ & 0.0004 (11) \\
\hline $\mathrm{C} 15$ & $0.0260(16)$ & $0.0253(17)$ & $0.0183(14)$ & $-0.0061(14)$ & $-0.0014(12)$ & $-0.0023(12)$ \\
\hline $\mathrm{C} 16$ & $0.0235(16)$ & $0.0272(17)$ & $0.0210(15)$ & $-0.0087(14)$ & $-0.0038(12)$ & $-0.0047(12)$ \\
\hline $\mathrm{C} 17$ & $0.0156(14)$ & $0.0205(15)$ & $0.0169(13)$ & $-0.0035(12)$ & $-0.0052(11)$ & $-0.0022(11)$ \\
\hline C18 & $0.0172(15)$ & 0.0243 (16) & $0.0214(14)$ & $-0.0026(13)$ & $-0.0040(12)$ & $-0.0002(12)$ \\
\hline C19 & 0.0163 (14) & $0.0184(14)$ & $0.0147(12)$ & $0.0009(12)$ & $0.0022(11)$ & $0.0001(10)$ \\
\hline $\mathrm{C} 20$ & $0.0312(18)$ & 0.0277 (17) & $0.0184(14)$ & $-0.0071(15)$ & $0.0062(13)$ & $-0.0071(12)$ \\
\hline $\mathrm{C} 21$ & $0.0202(15)$ & 0.0159 (14) & $0.0151(12)$ & $-0.0016(12)$ & -0.0055 & $-0.0002(10)$ \\
\hline $\mathrm{C} 22$ & $0.0161(14)$ & $0.0142(14)$ & 0.0215 (13) & $-0.0022(12)$ & $-0.0051(11)$ & $-0.0018(11)$ \\
\hline $\mathrm{C} 23$ & $0.0199(15)$ & $0.0183(14)$ & $0.0272(15)$ & $-0.0027(13)$ & $-0.0025(12)$ & $-0.0001(12)$ \\
\hline $\mathrm{C} 24$ & $0.0190(15)$ & $0.0223(16)$ & $0.0355(17)$ & $-0.0038(14)$ & $0.0013(13)$ & $-0.0082(13)$ \\
\hline $\mathrm{C} 25$ & $0.0169(15)$ & $0.0181(16)$ & $0.0476(19)$ & $0.0021(14)$ & $-0.0073(15)$ & $-0.0073(14)$ \\
\hline $\mathrm{C} 26$ & $0.0229(16)$ & $0.0172(16)$ & $0.0359(17)$ & $0.0014(14)$ & $-0.0126(14)$ & $0.0026(12)$ \\
\hline $\mathrm{C} 27$ & $0.0199(15)$ & $0.0198(15)$ & $0.0249(14)$ & $-0.0016(13)$ & $-0.0072(12)$ & $-0.0002(12)$ \\
\hline $\mathrm{C} 28$ & $0.0199(15)$ & $0.0181(15)$ & $0.0144(12)$ & $-0.0009(13)$ & $0.0002(11)$ & $-0.0020(10)$ \\
\hline $\mathrm{C} 29$ & $0.0257(16)$ & $0.0180(15)$ & $0.0129(12)$ & $-0.0036(13)$ & $0.0001(11)$ & $-0.0013(11)$ \\
\hline $\mathrm{C} 30$ & $0.0218(15)$ & $0.0204(15)$ & $0.0161(13)$ & $-0.0030(13)$ & $0.0020(11)$ & $-0.0016(11)$ \\
\hline C31 & $0.0285(17)$ & $0.0236(16)$ & $0.0245(15)$ & 0.0040 (14) & $-0.0038(13)$ & $0.0008(12)$ \\
\hline $\mathrm{C} 32$ & 0.0396 (19) & $0.0244(16)$ & 0.0197 (14) & $-0.0020(16)$ & $-0.0056(14)$ & $0.0091(12)$ \\
\hline C33 & 0.0295 (17) & 0.0302 (18) & $0.0203(15)$ & $-0.0072(15)$ & $0.0031(13)$ & $0.0058(12)$ \\
\hline C34 & $0.0210(15)$ & 0.0265 (17) & $0.0188(14)$ & $-0.0016(14)$ & $0.0032(11)$ & 0.0025 (12) \\
\hline
\end{tabular}


Geometric parameters $\left(\AA,{ }^{\circ}\right)$

\begin{tabular}{|c|c|c|c|}
\hline $\mathrm{O} 1-\mathrm{C} 5$ & $1.436(3)$ & $\mathrm{C} 11-\mathrm{H} 11 \mathrm{~B}$ & 0.9900 \\
\hline $\mathrm{O} 1-\mathrm{H} 1$ & $0.82(4)$ & $\mathrm{C} 12-\mathrm{C} 13$ & $1.347(4)$ \\
\hline $\mathrm{O} 2-\mathrm{C} 12$ & $1.372(4)$ & $\mathrm{C} 13-\mathrm{C} 15$ & $1.441(4)$ \\
\hline $\mathrm{O} 2-\mathrm{C} 16$ & $1.376(3)$ & $\mathrm{C} 13-\mathrm{C} 14$ & $1.496(4)$ \\
\hline O1W-HWA & $0.8501(14)$ & $\mathrm{C} 14-\mathrm{C} 20$ & $1.541(4)$ \\
\hline $\mathrm{O} 1 \mathrm{~W}-\mathrm{HWB}$ & $0.8501(14)$ & C14-H14 & 1.0000 \\
\hline $\mathrm{O} 3-\mathrm{C} 17$ & $1.337(3)$ & $\mathrm{C} 15-\mathrm{C} 16$ & $1.337(4)$ \\
\hline $\mathrm{O} 3-\mathrm{H} 3$ & $0.82(4)$ & $\mathrm{C} 15-\mathrm{H} 15$ & 0.9500 \\
\hline $\mathrm{O} 4-\mathrm{C} 17$ & $1.211(3)$ & C16-H16 & 0.9500 \\
\hline $\mathrm{O} 5-\mathrm{C} 21$ & $1.358(3)$ & $\mathrm{C} 18-\mathrm{H} 18 \mathrm{~A}$ & 0.9800 \\
\hline $\mathrm{O} 5-\mathrm{C} 6$ & $1.440(3)$ & C18-H18B & 0.9800 \\
\hline $\mathrm{O} 6-\mathrm{C} 21$ & $1.207(4)$ & $\mathrm{C} 18-\mathrm{H} 18 \mathrm{C}$ & 0.9800 \\
\hline $\mathrm{O} 7-\mathrm{C} 28$ & $1.350(3)$ & C19-H19A & 0.9800 \\
\hline $\mathrm{O} 7-\mathrm{C} 7$ & $1.455(3)$ & C19-H19B & 0.9800 \\
\hline $\mathrm{O} 8-\mathrm{C} 28$ & $1.206(4)$ & C19-H19C & 0.9800 \\
\hline $\mathrm{C} 1-\mathrm{C} 2$ & $1.522(4)$ & $\mathrm{C} 20-\mathrm{H} 20 \mathrm{~A}$ & 0.9800 \\
\hline $\mathrm{C} 1-\mathrm{C} 10$ & $1.551(4)$ & $\mathrm{C} 20-\mathrm{H} 20 \mathrm{~B}$ & 0.9800 \\
\hline $\mathrm{C} 1-\mathrm{H} 1 \mathrm{~A}$ & 0.9900 & $\mathrm{C} 20-\mathrm{H} 20 \mathrm{C}$ & 0.9800 \\
\hline $\mathrm{C} 1-\mathrm{H} 1 \mathrm{~B}$ & 0.9900 & $\mathrm{C} 21-\mathrm{C} 22$ & $1.484(4)$ \\
\hline $\mathrm{C} 2-\mathrm{C} 3$ & $1.522(4)$ & $\mathrm{C} 22-\mathrm{C} 23$ & $1.391(4)$ \\
\hline $\mathrm{C} 2-\mathrm{H} 2 \mathrm{~A}$ & 0.9900 & $\mathrm{C} 22-\mathrm{C} 27$ & $1.400(4)$ \\
\hline $\mathrm{C} 2-\mathrm{H} 2 \mathrm{~B}$ & 0.9900 & $\mathrm{C} 23-\mathrm{C} 24$ & $1.387(4)$ \\
\hline $\mathrm{C} 3-\mathrm{C} 4$ & $1.551(4)$ & $\mathrm{C} 23-\mathrm{H} 23$ & 0.9500 \\
\hline $\mathrm{C} 3-\mathrm{H} 3 \mathrm{~A}$ & 0.9900 & $\mathrm{C} 24-\mathrm{C} 25$ & $1.394(5)$ \\
\hline C $3-\mathrm{H} 3 \mathrm{~B}$ & 0.9900 & $\mathrm{C} 24-\mathrm{H} 24$ & 0.9500 \\
\hline $\mathrm{C} 4-\mathrm{C} 17$ & $1.524(4)$ & $\mathrm{C} 25-\mathrm{C} 26$ & $1.389(5)$ \\
\hline $\mathrm{C} 4-\mathrm{C} 18$ & $1.549(4)$ & $\mathrm{C} 25-\mathrm{H} 25$ & 0.9500 \\
\hline $\mathrm{C} 4-\mathrm{C} 5$ & $1.593(4)$ & $\mathrm{C} 26-\mathrm{C} 27$ & $1.384(4)$ \\
\hline $\mathrm{C} 5-\mathrm{C} 6$ & $1.552(4)$ & $\mathrm{C} 26-\mathrm{H} 26$ & 0.9500 \\
\hline $\mathrm{C} 5-\mathrm{C} 10$ & $1.572(4)$ & $\mathrm{C} 27-\mathrm{H} 27$ & 0.9500 \\
\hline $\mathrm{C} 6-\mathrm{C} 7$ & $1.532(4)$ & $\mathrm{C} 28-\mathrm{C} 29$ & $1.491(4)$ \\
\hline C6-H6 & 1.0000 & $\mathrm{C} 29-\mathrm{C} 30$ & $1.391(4)$ \\
\hline $\mathrm{C} 7-\mathrm{C} 8$ & $1.517(4)$ & $\mathrm{C} 29-\mathrm{C} 34$ & $1.398(4)$ \\
\hline $\mathrm{C} 7-\mathrm{H} 7$ & 1.0000 & $\mathrm{C} 30-\mathrm{C} 31$ & $1.390(4)$ \\
\hline $\mathrm{C} 8-\mathrm{C} 14$ & $1.559(4)$ & $\mathrm{C} 30-\mathrm{H} 30$ & 0.9500 \\
\hline $\mathrm{C} 8-\mathrm{C} 9$ & $1.562(3)$ & C31-C32 & $1.389(5)$ \\
\hline $\mathrm{C} 8-\mathrm{H} 8$ & 1.0000 & C $31-\mathrm{H} 31$ & 0.9500 \\
\hline C9- 111 & $1.546(4)$ & $\mathrm{C} 32-\mathrm{C} 33$ & $1.379(5)$ \\
\hline $\mathrm{C} 9-\mathrm{C} 10$ & $1.567(4)$ & $\mathrm{C} 32-\mathrm{H} 32$ & 0.9500 \\
\hline $\mathrm{C} 9-\mathrm{H} 9$ & 1.0000 & $\mathrm{C} 33-\mathrm{C} 34$ & $1.386(4)$ \\
\hline $\mathrm{C} 10-\mathrm{C} 19$ & $1.546(4)$ & C $33-\mathrm{H} 33$ & 0.9500 \\
\hline $\mathrm{C} 11-\mathrm{C} 12$ & $1.476(4)$ & C34-H34 & 0.9500 \\
\hline $\mathrm{C} 11-\mathrm{H} 11 \mathrm{~A}$ & 0.9900 & & \\
\hline $\mathrm{C} 5-\mathrm{O} 1-\mathrm{H} 1$ & 109.5 & $\mathrm{C} 15-\mathrm{C} 13-\mathrm{C} 14$ & $131.5(3)$ \\
\hline $\mathrm{C} 12-\mathrm{O} 2-\mathrm{C} 16$ & $105.7(2)$ & $\mathrm{C} 13-\mathrm{C} 14-\mathrm{C} 20$ & $110.0(2)$ \\
\hline
\end{tabular}




\begin{tabular}{|c|c|c|c|}
\hline $\mathrm{HWA}-\mathrm{O} 1 \mathrm{~W}-\mathrm{HWB}$ & $109.7(3)$ & $\mathrm{C} 13-\mathrm{C} 14-\mathrm{C} 8$ & $108.9(2)$ \\
\hline $\mathrm{C} 17-\mathrm{O} 3-\mathrm{H} 3$ & 109.5 & $\mathrm{C} 20-\mathrm{C} 14-\mathrm{C} 8$ & $114.8(2)$ \\
\hline $\mathrm{C} 21-\mathrm{O} 5-\mathrm{C} 6$ & $116.0(2)$ & $\mathrm{C} 13-\mathrm{C} 14-\mathrm{H} 14$ & 107.6 \\
\hline $\mathrm{C} 28-\mathrm{O} 7-\mathrm{C} 7$ & $116.2(2)$ & $\mathrm{C} 20-\mathrm{C} 14-\mathrm{H} 14$ & 107.6 \\
\hline $\mathrm{C} 2-\mathrm{C} 1-\mathrm{C} 10$ & $112.4(2)$ & $\mathrm{C} 8-\mathrm{C} 14-\mathrm{H} 14$ & 107.6 \\
\hline $\mathrm{C} 2-\mathrm{C} 1-\mathrm{H} 1 \mathrm{~A}$ & 109.1 & $\mathrm{C} 16-\mathrm{C} 15-\mathrm{C} 13$ & $106.3(3)$ \\
\hline $\mathrm{C} 10-\mathrm{C} 1-\mathrm{H} 1 \mathrm{~A}$ & 109.1 & $\mathrm{C} 16-\mathrm{C} 15-\mathrm{H} 15$ & 126.8 \\
\hline $\mathrm{C} 2-\mathrm{C} 1-\mathrm{H} 1 \mathrm{~B}$ & 109.1 & $\mathrm{C} 13-\mathrm{C} 15-\mathrm{H} 15$ & 126.8 \\
\hline $\mathrm{C} 10-\mathrm{C} 1-\mathrm{H} 1 \mathrm{~B}$ & 109.1 & $\mathrm{C} 15-\mathrm{C} 16-\mathrm{O} 2$ & $111.1(3)$ \\
\hline $\mathrm{H} 1 \mathrm{~A}-\mathrm{C} 1-\mathrm{H} 1 \mathrm{~B}$ & 107.9 & $\mathrm{C} 15-\mathrm{C} 16-\mathrm{H} 16$ & 124.5 \\
\hline $\mathrm{C} 1-\mathrm{C} 2-\mathrm{C} 3$ & $110.1(2)$ & $\mathrm{O} 2-\mathrm{C} 16-\mathrm{H} 16$ & 124.5 \\
\hline $\mathrm{C} 1-\mathrm{C} 2-\mathrm{H} 2 \mathrm{~A}$ & 109.6 & $\mathrm{O} 4-\mathrm{C} 17-\mathrm{O} 3$ & $121.2(3)$ \\
\hline $\mathrm{C} 3-\mathrm{C} 2-\mathrm{H} 2 \mathrm{~A}$ & 109.6 & $\mathrm{O} 4-\mathrm{C} 17-\mathrm{C} 4$ & $122.8(3)$ \\
\hline $\mathrm{C} 1-\mathrm{C} 2-\mathrm{H} 2 \mathrm{~B}$ & 109.6 & $\mathrm{O} 3-\mathrm{C} 17-\mathrm{C} 4$ & $115.7(2)$ \\
\hline $\mathrm{C} 3-\mathrm{C} 2-\mathrm{H} 2 \mathrm{~B}$ & 109.6 & $\mathrm{C} 4-\mathrm{C} 18-\mathrm{H} 18 \mathrm{~A}$ & 109.5 \\
\hline $\mathrm{H} 2 \mathrm{~A}-\mathrm{C} 2-\mathrm{H} 2 \mathrm{~B}$ & 108.2 & $\mathrm{C} 4-\mathrm{C} 18-\mathrm{H} 18 \mathrm{~B}$ & 109.5 \\
\hline $\mathrm{C} 2-\mathrm{C} 3-\mathrm{C} 4$ & $115.9(2)$ & $\mathrm{H} 18 \mathrm{~A}-\mathrm{C} 18-\mathrm{H} 18 \mathrm{~B}$ & 109.5 \\
\hline $\mathrm{C} 2-\mathrm{C} 3-\mathrm{H} 3 \mathrm{~A}$ & 108.3 & $\mathrm{C} 4-\mathrm{C} 18-\mathrm{H} 18 \mathrm{C}$ & 109.5 \\
\hline $\mathrm{C} 4-\mathrm{C} 3-\mathrm{H} 3 \mathrm{~A}$ & 108.3 & $\mathrm{H} 18 \mathrm{~A}-\mathrm{C} 18-\mathrm{H} 18 \mathrm{C}$ & 109.5 \\
\hline $\mathrm{C} 2-\mathrm{C} 3-\mathrm{H} 3 \mathrm{~B}$ & 108.3 & $\mathrm{H} 18 \mathrm{~B}-\mathrm{C} 18-\mathrm{H} 18 \mathrm{C}$ & 109.5 \\
\hline $\mathrm{C} 4-\mathrm{C} 3-\mathrm{H} 3 \mathrm{~B}$ & 108.3 & $\mathrm{C} 10-\mathrm{C} 19-\mathrm{H} 19 \mathrm{~A}$ & 109.5 \\
\hline $\mathrm{H} 3 \mathrm{~A}-\mathrm{C} 3-\mathrm{H} 3 \mathrm{~B}$ & 107.4 & $\mathrm{C} 10-\mathrm{C} 19-\mathrm{H} 19 \mathrm{~B}$ & 109.5 \\
\hline $\mathrm{C} 17-\mathrm{C} 4-\mathrm{C} 18$ & $102.6(2)$ & $\mathrm{H} 19 \mathrm{~A}-\mathrm{C} 19-\mathrm{H} 19 \mathrm{~B}$ & 109.5 \\
\hline $\mathrm{C} 17-\mathrm{C} 4-\mathrm{C} 3$ & $112.9(2)$ & $\mathrm{C} 10-\mathrm{C} 19-\mathrm{H} 19 \mathrm{C}$ & 109.5 \\
\hline $\mathrm{C} 18-\mathrm{C} 4-\mathrm{C} 3$ & $106.8(2)$ & $\mathrm{H} 19 \mathrm{~A}-\mathrm{C} 19-\mathrm{H} 19 \mathrm{C}$ & 109.5 \\
\hline $\mathrm{C} 17-\mathrm{C} 4-\mathrm{C} 5$ & $115.5(2)$ & $\mathrm{H} 19 \mathrm{~B}-\mathrm{C} 19-\mathrm{H} 19 \mathrm{C}$ & 109.5 \\
\hline $\mathrm{C} 18-\mathrm{C} 4-\mathrm{C} 5$ & $109.8(2)$ & $\mathrm{C} 14-\mathrm{C} 20-\mathrm{H} 20 \mathrm{~A}$ & 109.5 \\
\hline $\mathrm{C} 3-\mathrm{C} 4-\mathrm{C} 5$ & $108.8(2)$ & $\mathrm{C} 14-\mathrm{C} 20-\mathrm{H} 20 \mathrm{~B}$ & 109.5 \\
\hline $\mathrm{O} 1-\mathrm{C} 5-\mathrm{C} 6$ & $99.8(2)$ & $\mathrm{H} 20 \mathrm{~A}-\mathrm{C} 20-\mathrm{H} 20 \mathrm{~B}$ & 109.5 \\
\hline $\mathrm{O} 1-\mathrm{C} 5-\mathrm{C} 10$ & $109.7(2)$ & $\mathrm{C} 14-\mathrm{C} 20-\mathrm{H} 20 \mathrm{C}$ & 109.5 \\
\hline $\mathrm{C} 6-\mathrm{C} 5-\mathrm{C} 10$ & $111.2(2)$ & $\mathrm{H} 20 \mathrm{~A}-\mathrm{C} 20-\mathrm{H} 20 \mathrm{C}$ & 109.5 \\
\hline $\mathrm{O} 1-\mathrm{C} 5-\mathrm{C} 4$ & $104.6(2)$ & $\mathrm{H} 20 \mathrm{~B}-\mathrm{C} 20-\mathrm{H} 20 \mathrm{C}$ & 109.5 \\
\hline $\mathrm{C} 6-\mathrm{C} 5-\mathrm{C} 4$ & $115.2(2)$ & $\mathrm{O} 6-\mathrm{C} 21-\mathrm{O} 5$ & $124.0(3)$ \\
\hline $\mathrm{C} 10-\mathrm{C} 5-\mathrm{C} 4$ & $114.9(2)$ & $\mathrm{O} 6-\mathrm{C} 21-\mathrm{C} 22$ & $124.1(3)$ \\
\hline $\mathrm{O} 5-\mathrm{C} 6-\mathrm{C} 7$ & $108.4(2)$ & $\mathrm{O} 5-\mathrm{C} 21-\mathrm{C} 22$ & $111.9(2)$ \\
\hline $\mathrm{O} 5-\mathrm{C} 6-\mathrm{C} 5$ & $112.4(2)$ & $\mathrm{C} 23-\mathrm{C} 22-\mathrm{C} 27$ & $119.8(3)$ \\
\hline $\mathrm{C} 7-\mathrm{C} 6-\mathrm{C} 5$ & $109.3(2)$ & $\mathrm{C} 23-\mathrm{C} 22-\mathrm{C} 21$ & $122.1(3)$ \\
\hline $\mathrm{O} 5-\mathrm{C} 6-\mathrm{H} 6$ & 108.9 & $\mathrm{C} 27-\mathrm{C} 22-\mathrm{C} 21$ & $118.0(3)$ \\
\hline $\mathrm{C} 7-\mathrm{C} 6-\mathrm{H} 6$ & 108.9 & $\mathrm{C} 24-\mathrm{C} 23-\mathrm{C} 22$ & $120.1(3)$ \\
\hline $\mathrm{C} 5-\mathrm{C} 6-\mathrm{H} 6$ & 108.9 & $\mathrm{C} 24-\mathrm{C} 23-\mathrm{H} 23$ & 120.0 \\
\hline $\mathrm{O} 7-\mathrm{C} 7-\mathrm{C} 8$ & $107.4(2)$ & $\mathrm{C} 22-\mathrm{C} 23-\mathrm{H} 23$ & 120.0 \\
\hline $\mathrm{O} 7-\mathrm{C} 7-\mathrm{C} 6$ & $107.1(2)$ & $\mathrm{C} 23-\mathrm{C} 24-\mathrm{C} 25$ & $120.1(3)$ \\
\hline $\mathrm{C} 8-\mathrm{C} 7-\mathrm{C} 6$ & $114.4(2)$ & $\mathrm{C} 23-\mathrm{C} 24-\mathrm{H} 24$ & 120.0 \\
\hline $\mathrm{O} 7-\mathrm{C} 7-\mathrm{H} 7$ & 109.3 & $\mathrm{C} 25-\mathrm{C} 24-\mathrm{H} 24$ & 120.0 \\
\hline $\mathrm{C} 8-\mathrm{C} 7-\mathrm{H} 7$ & 109.3 & $\mathrm{C} 26-\mathrm{C} 25-\mathrm{C} 24$ & $119.8(3)$ \\
\hline $\mathrm{C} 6-\mathrm{C} 7-\mathrm{H} 7$ & 109.3 & $\mathrm{C} 26-\mathrm{C} 25-\mathrm{H} 25$ & 120.1 \\
\hline $\mathrm{C} 7-\mathrm{C} 8-\mathrm{C} 14$ & $109.9(2)$ & $\mathrm{C} 24-\mathrm{C} 25-\mathrm{H} 25$ & 120.1 \\
\hline $\mathrm{C} 7-\mathrm{C} 8-\mathrm{C} 9$ & $108.5(2)$ & $\mathrm{C} 27-\mathrm{C} 26-\mathrm{C} 25$ & $120.4(3)$ \\
\hline
\end{tabular}




\begin{tabular}{|c|c|c|c|}
\hline $\mathrm{C} 14-\mathrm{C} 8-\mathrm{C} 9$ & $113.5(2)$ & $\mathrm{C} 27-\mathrm{C} 26-\mathrm{H} 26$ & 119.8 \\
\hline $\mathrm{C} 7-\mathrm{C} 8-\mathrm{H} 8$ & 108.3 & $\mathrm{C} 25-\mathrm{C} 26-\mathrm{H} 26$ & 119.8 \\
\hline $\mathrm{C} 14-\mathrm{C} 8-\mathrm{H} 8$ & 108.3 & $\mathrm{C} 26-\mathrm{C} 27-\mathrm{C} 22$ & $119.8(3)$ \\
\hline $\mathrm{C} 9-\mathrm{C} 8-\mathrm{H} 8$ & 108.3 & $\mathrm{C} 26-\mathrm{C} 27-\mathrm{H} 27$ & 120.1 \\
\hline $\mathrm{C} 11-\mathrm{C} 9-\mathrm{C} 8$ & $112.5(2)$ & $\mathrm{C} 22-\mathrm{C} 27-\mathrm{H} 27$ & 120.1 \\
\hline $\mathrm{C} 11-\mathrm{C} 9-\mathrm{C} 10$ & $111.0(2)$ & $\mathrm{O} 8-\mathrm{C} 28-\mathrm{O} 7$ & $123.8(3)$ \\
\hline $\mathrm{C} 8-\mathrm{C} 9-\mathrm{C} 10$ & $112.2(2)$ & $\mathrm{O} 8-\mathrm{C} 28-\mathrm{C} 29$ & $125.0(3)$ \\
\hline $\mathrm{C} 11-\mathrm{C} 9-\mathrm{H} 9$ & 106.9 & $\mathrm{O} 7-\mathrm{C} 28-\mathrm{C} 29$ & $111.2(2)$ \\
\hline $\mathrm{C} 8-\mathrm{C} 9-\mathrm{H} 9$ & 106.9 & $\mathrm{C} 30-\mathrm{C} 29-\mathrm{C} 34$ & $120.1(3)$ \\
\hline $\mathrm{C} 10-\mathrm{C} 9-\mathrm{H} 9$ & 106.9 & $\mathrm{C} 30-\mathrm{C} 29-\mathrm{C} 28$ & $121.8(3)$ \\
\hline $\mathrm{C} 19-\mathrm{C} 10-\mathrm{C} 1$ & $107.4(2)$ & $\mathrm{C} 34-\mathrm{C} 29-\mathrm{C} 28$ & $118.0(3)$ \\
\hline $\mathrm{C} 19-\mathrm{C} 10-\mathrm{C} 9$ & $110.9(2)$ & $\mathrm{C} 31-\mathrm{C} 30-\mathrm{C} 29$ & $119.8(3)$ \\
\hline $\mathrm{C} 1-\mathrm{C} 10-\mathrm{C} 9$ & $108.8(2)$ & $\mathrm{C} 31-\mathrm{C} 30-\mathrm{H} 30$ & 120.1 \\
\hline $\mathrm{C} 19-\mathrm{C} 10-\mathrm{C} 5$ & $111.9(2)$ & $\mathrm{C} 29-\mathrm{C} 30-\mathrm{H} 30$ & 120.1 \\
\hline $\mathrm{C} 1-\mathrm{C} 10-\mathrm{C} 5$ & $108.9(2)$ & $\mathrm{C} 32-\mathrm{C} 31-\mathrm{C} 30$ & $119.7(3)$ \\
\hline $\mathrm{C} 9-\mathrm{C} 10-\mathrm{C} 5$ & $108.9(2)$ & $\mathrm{C} 32-\mathrm{C} 31-\mathrm{H} 31$ & 120.2 \\
\hline $\mathrm{C} 12-\mathrm{C} 11-\mathrm{C} 9$ & $110.9(2)$ & $\mathrm{C} 30-\mathrm{C} 31-\mathrm{H} 31$ & 120.2 \\
\hline $\mathrm{C} 12-\mathrm{C} 11-\mathrm{H} 11 \mathrm{~A}$ & 109.5 & $\mathrm{C} 33-\mathrm{C} 32-\mathrm{C} 31$ & $120.6(3)$ \\
\hline $\mathrm{C} 9-\mathrm{C} 11-\mathrm{H} 11 \mathrm{~A}$ & 109.5 & $\mathrm{C} 33-\mathrm{C} 32-\mathrm{H} 32$ & 119.7 \\
\hline $\mathrm{C} 12-\mathrm{C} 11-\mathrm{H} 11 \mathrm{~B}$ & 109.5 & $\mathrm{C} 31-\mathrm{C} 32-\mathrm{H} 32$ & 119.7 \\
\hline C9-C11-H11B & 109.5 & $\mathrm{C} 32-\mathrm{C} 33-\mathrm{C} 34$ & $120.2(3)$ \\
\hline $\mathrm{H} 11 \mathrm{~A}-\mathrm{C} 11-\mathrm{H} 11 \mathrm{~B}$ & 108.1 & $\mathrm{C} 32-\mathrm{C} 33-\mathrm{H} 33$ & 119.9 \\
\hline $\mathrm{C} 13-\mathrm{C} 12-\mathrm{O} 2$ & $110.9(3)$ & $\mathrm{C} 34-\mathrm{C} 33-\mathrm{H} 33$ & 119.9 \\
\hline $\mathrm{C} 13-\mathrm{C} 12-\mathrm{C} 11$ & $128.8(3)$ & $\mathrm{C} 33-\mathrm{C} 34-\mathrm{C} 29$ & $119.6(3)$ \\
\hline $\mathrm{O} 2-\mathrm{C} 12-\mathrm{C} 11$ & $120.3(2)$ & $\mathrm{C} 33-\mathrm{C} 34-\mathrm{H} 34$ & 120.2 \\
\hline $\mathrm{C} 12-\mathrm{C} 13-\mathrm{C} 15$ & $106.1(3)$ & $\mathrm{C} 29-\mathrm{C} 34-\mathrm{H} 34$ & 120.2 \\
\hline $\mathrm{C} 12-\mathrm{C} 13-\mathrm{C} 14$ & $122.4(3)$ & & \\
\hline $\mathrm{C} 10-\mathrm{C} 1-\mathrm{C} 2-\mathrm{C} 3$ & $-59.2(3)$ & $\mathrm{C} 10-\mathrm{C} 9-\mathrm{C} 11-\mathrm{C} 12$ & $160.0(2)$ \\
\hline $\mathrm{C} 1-\mathrm{C} 2-\mathrm{C} 3-\mathrm{C} 4$ & $56.0(3)$ & $\mathrm{C} 16-\mathrm{O} 2-\mathrm{C} 12-\mathrm{C} 13$ & $-0.1(3)$ \\
\hline $\mathrm{C} 2-\mathrm{C} 3-\mathrm{C} 4-\mathrm{C} 17$ & $80.5(3)$ & $\mathrm{C} 16-\mathrm{O} 2-\mathrm{C} 12-\mathrm{C} 11$ & $178.0(3)$ \\
\hline $\mathrm{C} 2-\mathrm{C} 3-\mathrm{C} 4-\mathrm{C} 18$ & $-167.5(2)$ & $\mathrm{C} 9-\mathrm{C} 11-\mathrm{C} 12-\mathrm{C} 13$ & $-8.6(4)$ \\
\hline $\mathrm{C} 2-\mathrm{C} 3-\mathrm{C} 4-\mathrm{C} 5$ & $-49.1(3)$ & $\mathrm{C} 9-\mathrm{C} 11-\mathrm{C} 12-\mathrm{O} 2$ & $173.6(2)$ \\
\hline $\mathrm{C} 17-\mathrm{C} 4-\mathrm{C} 5-\mathrm{O} 1$ & $158.7(2)$ & $\mathrm{O} 2-\mathrm{C} 12-\mathrm{C} 13-\mathrm{C} 15$ & $0.0(3)$ \\
\hline $\mathrm{C} 18-\mathrm{C} 4-\mathrm{C} 5-\mathrm{O} 1$ & $43.5(3)$ & $\mathrm{C} 11-\mathrm{C} 12-\mathrm{C} 13-\mathrm{C} 15$ & $-178.0(3)$ \\
\hline $\mathrm{C} 3-\mathrm{C} 4-\mathrm{C} 5-\mathrm{O} 1$ & $-73.1(3)$ & $\mathrm{O} 2-\mathrm{C} 12-\mathrm{C} 13-\mathrm{C} 14$ & $-178.6(2)$ \\
\hline $\mathrm{C} 17-\mathrm{C} 4-\mathrm{C} 5-\mathrm{C} 6$ & $50.3(3)$ & $\mathrm{C} 11-\mathrm{C} 12-\mathrm{C} 13-\mathrm{C} 14$ & $3.4(5)$ \\
\hline $\mathrm{C} 18-\mathrm{C} 4-\mathrm{C} 5-\mathrm{C} 6$ & $-65.0(3)$ & $\mathrm{C} 12-\mathrm{C} 13-\mathrm{C} 14-\mathrm{C} 20$ & $104.1(3)$ \\
\hline $\mathrm{C} 3-\mathrm{C} 4-\mathrm{C} 5-\mathrm{C} 6$ & $178.5(2)$ & $\mathrm{C} 15-\mathrm{C} 13-\mathrm{C} 14-\mathrm{C} 20$ & $-74.1(4)$ \\
\hline $\mathrm{C} 17-\mathrm{C} 4-\mathrm{C} 5-\mathrm{C} 10$ & $-80.9(3)$ & $\mathrm{C} 12-\mathrm{C} 13-\mathrm{C} 14-\mathrm{C} 8$ & $-22.5(4)$ \\
\hline $\mathrm{C} 18-\mathrm{C} 4-\mathrm{C} 5-\mathrm{C} 10$ & $163.8(2)$ & $\mathrm{C} 15-\mathrm{C} 13-\mathrm{C} 14-\mathrm{C} 8$ & $159.2(3)$ \\
\hline $\mathrm{C} 3-\mathrm{C} 4-\mathrm{C} 5-\mathrm{C} 10$ & $47.3(3)$ & $\mathrm{C} 7-\mathrm{C} 8-\mathrm{C} 14-\mathrm{C} 13$ & $169.4(2)$ \\
\hline $\mathrm{C} 21-\mathrm{O} 5-\mathrm{C} 6-\mathrm{C} 7$ & $-99.4(2)$ & $\mathrm{C} 9-\mathrm{C} 8-\mathrm{C} 14-\mathrm{C} 13$ & $47.7(3)$ \\
\hline $\mathrm{C} 21-\mathrm{O} 5-\mathrm{C} 6-\mathrm{C} 5$ & $139.7(2)$ & $\mathrm{C} 7-\mathrm{C} 8-\mathrm{C} 14-\mathrm{C} 20$ & $45.6(3)$ \\
\hline $\mathrm{O} 1-\mathrm{C} 5-\mathrm{C} 6-\mathrm{O} 5$ & $179.7(2)$ & $\mathrm{C} 9-\mathrm{C} 8-\mathrm{C} 14-\mathrm{C} 20$ & $-76.1(3)$ \\
\hline $\mathrm{C} 10-\mathrm{C} 5-\mathrm{C} 6-\mathrm{O} 5$ & $64.0(3)$ & $\mathrm{C} 12-\mathrm{C} 13-\mathrm{C} 15-\mathrm{C} 16$ & $0.2(4)$ \\
\hline $\mathrm{C} 4-\mathrm{C} 5-\mathrm{C} 6-\mathrm{O} 5$ & $-69.0(3)$ & $\mathrm{C} 14-\mathrm{C} 13-\mathrm{C} 15-\mathrm{C} 16$ & $178.6(3)$ \\
\hline $\mathrm{O} 1-\mathrm{C} 5-\mathrm{C} 6-\mathrm{C} 7$ & $59.3(3)$ & $\mathrm{C} 13-\mathrm{C} 15-\mathrm{C} 16-\mathrm{O} 2$ & $-0.3(4)$ \\
\hline
\end{tabular}




$$
\begin{aligned}
& \mathrm{C} 10-\mathrm{C} 5-\mathrm{C} 6-\mathrm{C} 7 \\
& \mathrm{C} 4-\mathrm{C} 5-\mathrm{C} 6-\mathrm{C} 7 \\
& \mathrm{C} 28-\mathrm{O} 7-\mathrm{C} 7-\mathrm{C} 8 \\
& \mathrm{C} 28-\mathrm{O} 7-\mathrm{C} 7-\mathrm{C} 6 \\
& \mathrm{O} 5-\mathrm{C} 6-\mathrm{C} 7-\mathrm{O} 7 \\
& \mathrm{C} 5-\mathrm{C} 6-\mathrm{C} 7-\mathrm{O} 7 \\
& \mathrm{O} 5-\mathrm{C} 6-\mathrm{C} 7-\mathrm{C} 8 \\
& \mathrm{C} 5-\mathrm{C} 6-\mathrm{C} 7-\mathrm{C} 8 \\
& \mathrm{O} 7-\mathrm{C} 7-\mathrm{C} 8-\mathrm{C} 14 \\
& \mathrm{C} 6-\mathrm{C} 7-\mathrm{C} 8-\mathrm{C} 14 \\
& \mathrm{O} 7-\mathrm{C} 7-\mathrm{C} 8-\mathrm{C} 9 \\
& \mathrm{C} 6-\mathrm{C} 7-\mathrm{C} 8-\mathrm{C} 9 \\
& \mathrm{C} 7-\mathrm{C} 8-\mathrm{C} 9-\mathrm{C} 11 \\
& \mathrm{C} 14-\mathrm{C} 8-\mathrm{C} 9-\mathrm{C} 11 \\
& \mathrm{C} 7-\mathrm{C} 8-\mathrm{C} 9-\mathrm{C} 10 \\
& \mathrm{C} 14-\mathrm{C} 8-\mathrm{C} 9-\mathrm{C} 10 \\
& \mathrm{C} 2-\mathrm{C} 1-\mathrm{C} 10-\mathrm{C} 19 \\
& \mathrm{C} 2-\mathrm{C} 1-\mathrm{C} 10-\mathrm{C} 9 \\
& \mathrm{C} 2-\mathrm{C} 1-\mathrm{C} 10-\mathrm{C} 5 \\
& \mathrm{C} 11-\mathrm{C} 9-\mathrm{C} 10-\mathrm{C} 19 \\
& \mathrm{C} 8-\mathrm{C} 9-\mathrm{C} 10-\mathrm{C} 19 \\
& \mathrm{C} 11-\mathrm{C} 9-\mathrm{C} 10-\mathrm{C} 1 \\
& \mathrm{C} 8-\mathrm{C} 9-\mathrm{C} 10-\mathrm{C} 1 \\
& \mathrm{C} 11-\mathrm{C} 9-\mathrm{C} 10-\mathrm{C} 5 \\
& \mathrm{C} 8-\mathrm{C} 9-\mathrm{C} 10-\mathrm{C} 5 \\
& \mathrm{O} 1-\mathrm{C} 5-\mathrm{C} 10-\mathrm{C} 19 \\
& \text { C6- C5-C10-C19 } \\
& \mathrm{C} 4-\mathrm{C} 5-\mathrm{C} 10-\mathrm{C} 19 \\
& \mathrm{O} 1-\mathrm{C} 5-\mathrm{C} 10-\mathrm{C} 1 \\
& \mathrm{C} 6-\mathrm{C} 5-\mathrm{C} 10-\mathrm{C} 1 \\
& \mathrm{C} 4-\mathrm{C} 5-\mathrm{C} 10-\mathrm{C} 1 \\
& \mathrm{O} 1-\mathrm{C} 5-\mathrm{C} 10-\mathrm{C} 9 \\
& \mathrm{C} 6-\mathrm{C} 5-\mathrm{C} 10-\mathrm{C} 9 \\
& \mathrm{C} 4-\mathrm{C} 5-\mathrm{C} 10-\mathrm{C} 9 \\
& \mathrm{C} 8-\mathrm{C} 9-\mathrm{C} 11-\mathrm{C} 12
\end{aligned}
$$

$$
\begin{aligned}
& 0.3(3) \\
& 48.5(3) \\
& 163.0(3) \\
& -70.8(4) \\
& -126.3(2) \\
& -11.8(4) \\
& 114.4(3) \\
& 2.4(4) \\
& -177.6(2) \\
& -150.1(3) \\
& 30.0(4) \\
& 26.0(4) \\
& -153.9(2) \\
& -1.8(4) \\
& 174.2(3) \\
& 1.5(4) \\
& 0.1(5) \\
& -1.5(5) \\
& 1.2(4) \\
& 0.4(4) \\
& -175.7(3) \\
& 11.8(4) \\
& -167.4(2) \\
& -175.4(3) \\
& 3.7(4) \\
& 2.2(4) \\
& -178.7(3) \\
& -1.3(4) \\
& 176.3(3) \\
& 0.4(4) \\
& 0.4(5) \\
& -0.3(5) \\
& -0.5(5) \\
& 1.3(4) \\
& -176.3(3) \\
&
\end{aligned}
$$

Hydrogen-bond geometry $(\AA, \stackrel{\circ}{)}$

$\mathrm{Cg} 1$ and $\mathrm{Cg} 2$ are the centroids of the $\mathrm{C} 29-\mathrm{C} 34$ and $\mathrm{C} 22-\mathrm{C} 27$ rings, respectively.

\begin{tabular}{lllll}
\hline$D-\mathrm{H} \cdots A$ & $D-\mathrm{H}$ & $\mathrm{H} \cdots A$ & $D \cdots A$ & $D-\mathrm{H} \cdots A$ \\
\hline $\mathrm{O} 1-\mathrm{H} 1 \cdots \mathrm{O} 4^{\mathrm{i}}$ & $0.82(4)$ & $2.01(4)$ & $2.654(3)$ & $134(4)$ \\
$\mathrm{O} 1 W-\mathrm{H} W A \cdots \mathrm{O} 1$ & $0.85(2)$ & $2.03(3)$ & $2.838(3)$ & $159(2)$ \\
$\mathrm{O} 1 W-\mathrm{H} W B \cdots \mathrm{O} 8$ & $0.85(2)$ & $2.27(3)$ & $3.062(3)$ & $154(2)$ \\
$\mathrm{O} 3-\mathrm{H} 3 \cdots \mathrm{O} 1 W^{\mathrm{ii}}$ & $0.83(4)$ & $1.86(4)$ & $2.680(3)$ & $174(4)$ \\
$\mathrm{C} 19-\mathrm{H} 19 B \cdots \mathrm{O} 3$ & 0.98 & 2.51 & $3.445(3)$ & 159
\end{tabular}


supporting information

$\begin{array}{llllr}\mathrm{C} 1-\mathrm{H} 1 A \cdots C g 1^{\mathrm{iii}} & 0.99 & 2.98 & 3.910(3) & 157 \\ \mathrm{C} 34-\mathrm{H} 34 \cdots C g 2^{\mathrm{iv}} & 0.95 & 2.85 & 3.655(3) & 143\end{array}$

Symmetry codes: (i) $-x+1, y-1 / 2,-z+3 / 2$; (ii) $-x+1, y+1 / 2,-z+3 / 2$; (iii) $-x+1 / 2,-y+1, z+1 / 2$; (iv) $x+1 / 2,-y+3 / 2,-z+1$. 